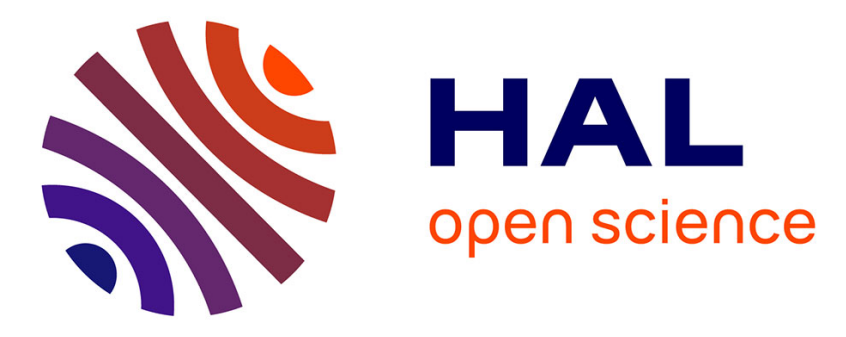

\title{
Fluctuation-induced forces on an atom near a photonic topological material
}

\author{
Mario Silveirinha, S. Ali Hassani Gangaraj, George W. Hanson, Mauro \\ Antezza
}

\section{- To cite this version:}

Mario Silveirinha, S. Ali Hassani Gangaraj, George W. Hanson, Mauro Antezza. Fluctuation-induced forces on an atom near a photonic topological material. Physical Review A : Atomic, molecular, and optical physics [1990-2015], 2018, 97, pp.022509. 10.1103/PhysRevA.97.022509 . hal-01714025

\section{HAL Id: hal-01714025 \\ https://hal.science/hal-01714025}

Submitted on 13 Oct 2020

HAL is a multi-disciplinary open access archive for the deposit and dissemination of scientific research documents, whether they are published or not. The documents may come from teaching and research institutions in France or abroad, or from public or private research centers.
L'archive ouverte pluridisciplinaire HAL, est destinée au dépôt et à la diffusion de documents scientifiques de niveau recherche, publiés ou non, émanant des établissements d'enseignement et de recherche français ou étrangers, des laboratoires publics ou privés. 


\title{
Fluctuation-induced forces on an atom near a photonic topological material
}

\author{
Mário G. Silveirinha, ${ }^{1,2, *}$ S. Ali Hassani Gangaraj, ${ }^{3, \dagger}$ George W. Hanson, ${ }^{4, \ddagger}$ and Mauro Antezza ${ }^{2,5,8}$ \\ ${ }^{1}$ Instituto Superior Técnico, University of Lisbon and Instituto de Telecomunicações, Torre Norte, Av. Rovisco Pais 1, \\ P-1049-001 Lisbon, Portugal \\ ${ }^{2}$ Laboratoire Charles Coulomb (L2C), UMR 5221 CNRS-Université de Montpellier, F-34095 Montpellier, France \\ ${ }^{3}$ School of Electrical and Computer Engineering, Cornell University, Ithaca, New York 14853, USA \\ ${ }^{4}$ Department of Electrical Engineering, University of Wisconsin-Milwaukee, 3200 N. Cramer St., Milwaukee, Wisconsin 53211, USA \\ ${ }^{5}$ Institut Universitaire de France, 1 Rue Descartes, F-75231 Paris Cedex 05, France
}

(Received 13 November 2017; published 20 February 2018)

\begin{abstract}
We theoretically study the Casimir-Polder force on an atom in an arbitrary initial state in a rather general electromagnetic environment wherein the materials may have a nonreciprocal bianisotropic dispersive response. It is shown that under the Markov approximation the force has resonant and nonresonant contributions. We obtain explicit expressions for the optical force both in terms of the system Green function and of the electromagnetic modes. We apply the theory to the particular case wherein a two-level system interacts with a topological gyrotropic material, showing that the nonreciprocity enables exotic light-matter interactions and the opportunity to sculpt and tune the Casimir-Polder forces on the nanoscale. With a quasistatic approximation, we obtain a simple analytical expression for the optical force and unveil the crucial role of surface plasmons in fluctuation-induced forces. Finally, we derive the Green function for a gyrotropic material half-space in terms of a Sommerfeld integral.
\end{abstract}

DOI: 10.1103/PhysRevA.97.022509

\section{INTRODUCTION}

The Casimir-Polder force acting on atoms located close to the surface of a material body is of longstanding and current interest [1-17], and is of considerable practical importance in a variety of physical, biological, and chemical processes. For planar surfaces, the normal component of the force has been extensively investigated both theoretically and experimentally [18]. There is a vast literature on theoretical methods to calculate the force when the material structures are conventional isotropic dispersive dielectrics $[3,5-12,15]$. Furthermore, the Casimir-Lifshitz interactions between two macroscopic bodies with exotic electromagnetic responses have also been discussed in a variety of scenarios [17,19-28], but the majority of the works consider planar geometries and that the system is in the ground state. Indeed, it seems that the Casimir-Polder interaction between a neutral atom and a generic environment with a complex (e.g., gyrotropic or bianisotropic) electromagnetic response has not been fully addressed so far in the literature.

In this article, motivated by the recent interest in nonreciprocal photonic platforms with topological properties [29-38], we develop a theoretical formalism to characterize the Casimir-Polder force acting on an atom prepared in an arbitrary initial state in the vicinity of an arbitrary possibly bianisotropic, inhomogeneous, and nonreciprocal dispersive system. In the general case, the optical force is written in terms

\footnotetext{
*mario.silveirinha@co.it.pt

†ali.gangaraj@gmail.com

${ }^{\ddagger}$ george@uwm.edu

§mauro.antezza@umontpellier.fr
}

of the system Green function. Interestingly, we show that in the limit of vanishing material loss the quantum force may be written as well in terms of the electromagnetic modes of the system.

We apply the developed formalism to a two-level atom placed in the vicinity of a topological gyrotropic material, e.g., a magnetically biased plasma [34-36]. Based on a simple quasistatic approximation, we obtain explicit formulas for the fluctuation-induced force and highlight how by tuning the strength of the nonreciprocal response it is possible to tailor the amplitude of the lateral and normal components of the optical force. Furthermore, our analysis reveals that the fluctuation-induced force is largely determined by the surface plasmon polaritons (SPPs). The "exact" quantum force is numerically computed using the Green function for an gyrotropic half-space, which is also derived here. It is shown that the quasistatic approximation agrees rather well with the result obtained with the exact Green function. Moreover, in [39], the developed theory is used to show that excited atoms may experience nonzero spontaneous lateral forces when near a photonic topological insulator. Unlike previous studies [16,4043], in a topological system the sign of the lateral optical force may be polarization and orientation independent and is tunable [39].

The article is organized as follows. In Sec. II, we derive the vacuum fluctuation-induced Casimir-Polder force acting on an atom in a generic electromagnetic environment. The effect of thermal fluctuations is neglected and the Markov approximation is used to solve the Heisenberg equations. For simplicity, the analysis is focused on two-level systems, but we provide also the expression of the force for the case of multilevel atoms. In Sec. III, we consider the scenario wherein the electromagnetic environment is a topological material half- 
space. Assuming that the material has a gyrotropic response (magnetized plasma), we characterize the edge (SPP) modes supported by the system and obtain closed-form expressions for the Casimir-Polder force under a quasistatic approximation. In Sec. V we present a numerical study that illustrates how by controlling the strength of the biasing magnetic field it is possible to tailor the amplitude and in some cases also the sign of the Casimir-Polder force. Finally, a short summary of the main findings is given in Sec. VI.

\section{OPTICAL FORCE}

In this section, we prove that in a rather general context the expectation of the optical force acting on a two-level atom can be decomposed into a resonant term $\left(\mathscr{F}_{R, i}\right)$ and a nonresonant term $\left(\mathscr{F}_{C, i}\right)$ as

$$
\mathscr{F}_{i}(t)=\left\langle\hat{\mathscr{F}}_{i}\right\rangle=\rho_{e e}(t) \mathscr{F}_{R, i}+\left(1-2 \rho_{e e}(t)\right) \mathscr{F}_{C, i},
$$

with $\rho_{e e}(t)$ the probability of the atom being in the excited state. The resonant component of the force is determined by the system Green function $\mathbf{G}(\mathrm{a} 6 \times 6$ tensor; see Appendix A) evaluated at the two-level atom transition frequency $\left(\omega_{0}\right)$,

$$
\mathscr{F}_{R, i}=2 \operatorname{Re}\left\{\left.\tilde{\gamma}^{*} \cdot\left(-i \omega \partial_{i} \mathbf{G}\left(\mathbf{r}, \mathbf{r}_{0} ; \omega\right)\right)\right|_{\substack{\omega=\omega_{0}+i 0^{+} \\ \mathbf{r}=\mathbf{r}_{0}}} \cdot \tilde{\gamma}\right\} .
$$

Here, $\partial_{i}=\hat{\mathbf{u}}_{i} \cdot \nabla_{\mathbf{r}}$ represents the spatial derivative along the $i$ th space direction, $\tilde{\gamma}=\left[\begin{array}{ll}\gamma & 0\end{array}\right]^{T}$ is a six-vector and $\gamma$ is the dipole transition matrix element. The atom coordinates are determined by the vector $\mathbf{r}_{0}$. It is assumed that the atom is surrounded by a vacuum (free-space) in its immediate vicinity.

The nonresonant component of the force gives the CasimirPolder force due to the zero-point fluctuations $\mathscr{F}_{C}=-\nabla_{\mathbf{r}_{0}} \mathscr{E}_{C}$, and depends on the interaction Casimir energy,

$$
\mathscr{E}_{C}=\frac{-1}{4 \pi} \int_{-\infty}^{\infty} d \xi \operatorname{tr}\left(\tilde{\alpha}(i \xi) \cdot(-i \omega \mathbf{G})_{\omega=i \xi}\right) .
$$

In the above, "tr" stands for the trace of a matrix and $\tilde{\alpha}(\omega)=$ $\left(\frac{1}{\omega_{0}-\omega} \tilde{\gamma} \tilde{\gamma}^{*}+\frac{1}{\omega_{0}+\omega} \tilde{\gamma}^{*} \tilde{\gamma}\right)$, so that $\alpha_{i j}=\tilde{\alpha}_{i j} /\left(\hbar \varepsilon_{0}\right)$ represents the semiclassical ground-state electric polarizability of the two-level system $(i, j=1,2,3)$ [44]. The Green function is evaluated at imaginary frequencies $(\omega=i \xi)$ with identical observation and source points, $\mathbf{r}=\mathbf{r}^{\prime}=\mathbf{r}_{0}$. The result (1) holds in the low-temperature limit: $k_{B} T \ll \hbar \omega_{0}$ and $d \ll \lambda_{T}$, with $d$ the minimum distance between the atom and the macroscopic bodies and $\lambda_{T}=h c / k_{B} T$ the thermal wavelength. The Green function can be generally decomposed as $\mathbf{G}=\mathbf{G}_{0}+\mathbf{G}_{s}$, with $\mathbf{G}_{0}$ the free-space Green function corresponding to the situation wherein the atom resides in a vacuum. Due to symmetry reasons, $\mathbf{G}_{0}$ cannot contribute to the force in the electric dipole approximation. Hence, in Eqs. (2) and (3) the Green function can be replaced by its "scattering part" $\mathbf{G}_{s}$, which is free of singularities when $\mathbf{r}=\mathbf{r}^{\prime}=\mathbf{r}_{0}$.

For a two-level system the excited state probability is $\rho_{e e}(t)=\rho_{e e}(0) e^{-\Gamma_{e g} t}$ with

$$
\Gamma_{e g}=\frac{2}{\hbar} \operatorname{Im}\left\{\left.\tilde{\gamma}^{*} \cdot(-i \omega \mathbf{G})\right|_{\omega=\omega_{0}} \cdot \tilde{\gamma}\right\}
$$

the standard spontaneous emission decay rate [45].

Note that in the electric dipole approximation the force only depends on the "electric part" of the Green function $\mathbf{G}_{\mathrm{EE}}$, defined as in Eq. (A1) of Appendix A (a $3 \times 3$ tensor). For standard dielectric media (with a trivial magnetic response and vanishing magnetoelectric tensors) $\mathbf{G}_{\mathrm{EE}}$ is related to the more conventional Green function definition $\mathcal{G}$ of Refs. [12,15] as $\mathbf{G}_{\mathrm{EE}}=i \omega \mu_{0} \mathcal{G}$.

\section{A. Modal expansion}

To begin with, we obtain a formula for the optical force in terms of the natural modes of oscillation of the electromagnetic field. Hence, in this section we consider the limit of vanishing material loss. For convenience, we adopt six-vector notations so that the quantized electromagnetic fields are denoted by the six-vector operator $\hat{\mathbf{F}}=(\hat{\mathbf{E}} \hat{\mathbf{H}})^{\mathrm{T}}$. The hat indicates that a given symbol represents an operator.

From the correspondence principle, the optical force operator is (electric dipole approximation) [46]

$$
\hat{\mathscr{F}}_{j}=\hat{\mathbf{p}}_{g} \cdot \frac{\partial}{\partial j} \hat{\mathbf{F}}, \quad j=x, y, z,
$$

where $\hat{\mathbf{p}}_{g}=(\hat{\mathbf{p}} \hat{\mathbf{0}})^{\mathrm{T}}$ is a generalized dipole moment operator and $\hat{\mathbf{p}}$ is the standard electric dipole operator for the two-level atom. The quantized electromagnetic field in a generic inhomogeneous and dispersive material platform can be written in terms of positive and negative frequency components $\hat{\mathbf{F}}=$ $\hat{\mathbf{F}}_{-}+\hat{\mathbf{F}}_{+}$with $\hat{\mathbf{F}}_{+}=\hat{\mathbf{F}}_{-}^{\dagger}$, and [45,47-49]

$$
\hat{\mathbf{F}}_{-}(\mathbf{r}, t)=\sum_{\omega_{n \mathbf{k}}>0} \sqrt{\frac{\hbar \omega_{n \mathbf{k}}}{2}} \mathbf{F}_{n \mathbf{k}}(\mathbf{r}) \hat{a}_{n \mathbf{k}}(t) .
$$

In the above, $\mathbf{F}_{n \mathbf{k}}(\mathbf{r})$ represents a generic cavity mode with oscillation frequency $\omega_{n \mathbf{k}}$, and $\hat{a}_{n \mathbf{k}}(t)$ is the corresponding bosonic operator satisfying $\left[\hat{a}_{n \mathbf{k}}, \hat{a}_{n \mathbf{k}}^{\dagger}\right]=1$. The electromagnetic modes $\mathbf{F}_{n \mathbf{k}}$ are normalized as [45,47-49]

$$
\frac{1}{2} \int d^{3} \mathbf{r} \mathbf{F}_{n \mathbf{k}}^{*} \cdot \frac{\partial(\omega \mathbf{M})}{\partial \omega} \cdot \mathbf{F}_{n \mathbf{k}}=1,
$$

where $\mathbf{M}=\mathbf{M}(\mathbf{r}, \omega)$ is the $6 \times 6$ material matrix that describes the electromagnetic properties of the environment. It relates the classical $\mathbf{D}$ and $\mathbf{B}$ fields with the classical $\mathbf{E}$ and $\mathbf{H}$ fields. For a generic bianisotropic (eventually nonreciprocal) material it is of the form,

$$
\mathbf{M}(\mathbf{r}, \omega)=\left(\begin{array}{cc}
\varepsilon & \frac{1}{c} \xi \\
\frac{1}{c} \zeta & \mu
\end{array}\right) .
$$

The $3 \times 3$ tensors $\boldsymbol{\varepsilon}$ and $\boldsymbol{\mu}$ represent the permittivity and permeability, and the tensors $\xi$ and $\zeta$ determine the magnetoelectric response.

Using normal ordering of the field operators, the expectation of the force can be written as

$$
\mathscr{F}_{j}=\left\langle\hat{\mathscr{F}}_{j}\right\rangle=2 \operatorname{Re}\left\langle\hat{\mathbf{p}}_{g} \cdot \partial_{j} \hat{\mathbf{F}}_{-}\right\rangle .
$$

In the above, the field is evaluated at $\mathbf{r}=\mathbf{r}_{0}$, the position of the atom, and the Heisenberg picture is implicit.

The total Hamiltonian of the system is

$$
\begin{aligned}
\hat{H}= & \hbar \omega_{0} \hat{\sigma}_{+} \hat{\sigma}_{-}+\sum_{\omega_{n \mathbf{k}}>0} \frac{\hbar \omega_{n \mathbf{k}}}{2}\left(\hat{a}_{n \mathbf{k}} \hat{a}_{n \mathbf{k}}^{\dagger}+\hat{a}_{n \mathbf{k}}^{\dagger} \hat{a}_{n \mathbf{k}}\right) \\
& -\hat{\mathbf{p}} \cdot \hat{\mathbf{E}}\left(\mathbf{r}_{0}\right)
\end{aligned}
$$


where the last term is the interaction Hamiltonian $\hat{H}_{\text {int }}$. With $\hat{\mathbf{p}}=\boldsymbol{\gamma}^{*} \hat{\sigma}_{+}+\boldsymbol{\gamma} \hat{\sigma}_{-}$, it can be written as

$$
\begin{aligned}
\hat{H}_{\text {int }} & =-\left(\boldsymbol{\gamma}^{*} \hat{\sigma}_{+}+\boldsymbol{\gamma} \hat{\sigma}_{-}\right) \cdot \hat{\mathbf{E}}\left(\mathbf{r}_{0}\right) \\
& =-\left(\tilde{\boldsymbol{\gamma}}^{*} \hat{\sigma}_{+}+\tilde{\boldsymbol{\gamma}} \hat{\sigma}_{-}\right) \cdot \hat{\mathbf{F}}\left(\mathbf{r}_{0}\right),
\end{aligned}
$$

where $\hat{\sigma}_{ \pm}$are the atom raising and lowering operators.

Using the Heisenberg equation of motion, $\partial_{t} \hat{a}_{n \mathbf{k}}=$ $i \hbar^{-1}\left[\hat{H}, \hat{a}_{n \mathbf{k}}\right]$, it follows that

$$
\frac{\partial \hat{a}_{n \mathbf{k}}}{\partial t}=-i \omega_{n \mathbf{k}} \hat{a}_{n \mathbf{k}}+\frac{i}{\hbar} \hat{\mathbf{p}} \cdot \sqrt{\frac{\hbar \omega_{n \mathbf{k}}}{2}} \mathbf{E}_{n \mathbf{k}}^{*}\left(\mathbf{r}_{0}\right) .
$$

By integrating the differential equation one obtains [45]

$$
\begin{aligned}
\hat{a}_{n \mathbf{k}}(t)= & \hat{a}_{n \mathbf{k}} e^{-i \omega_{n \mathbf{k}} t}+\int \frac{i}{\hbar} \hat{\mathbf{p}}\left(t^{\prime}\right) \cdot \sqrt{\frac{\hbar \omega_{n \mathbf{k}}}{2}} \mathbf{E}_{n \mathbf{k}}^{*}\left(\mathbf{r}_{0}\right) u\left(t-t^{\prime}\right) \\
& \times e^{-i \omega_{n \mathbf{k}}\left(t-t^{\prime}\right)} d t^{\prime} .
\end{aligned}
$$

Using the Markov approximation and

$$
\begin{aligned}
& \int_{t_{0}}^{t} u\left(t-t^{\prime}\right) e^{-i\left(\omega_{n \mathbf{k}}-\omega_{0}\right)\left(t-t^{\prime}\right)} d t^{\prime} \\
& \quad \approx \pi \delta\left(\omega_{n \mathbf{k}}-\omega_{0}\right)+\mathrm{P} \frac{1}{i\left(\omega_{n \mathbf{k}}-\omega_{0}\right)}
\end{aligned}
$$

for an interaction that starts at $t_{0} \rightarrow-\infty$, it is found that

$$
\begin{aligned}
\hat{a}_{n \mathbf{k}}(t) \approx & \hat{a}_{n \mathbf{k}} e^{-i \omega_{n \mathbf{k}} t} \\
& +\sqrt{\frac{\omega_{n \mathbf{k}}}{2 \hbar}} \tilde{\gamma} \cdot \mathbf{F}_{n \mathbf{k}}^{*} \hat{\sigma}_{-}(t) \frac{1}{\omega_{n \mathbf{k}}-\omega_{0}-i 0^{+}} \\
& +\sqrt{\frac{\omega_{n \mathbf{k}}}{2 \hbar}} \tilde{\gamma}^{*} \cdot \mathbf{F}_{n \mathbf{k}}^{*} \hat{\sigma}_{+}(t) \frac{1}{\omega_{n \mathbf{k}}+\omega_{0}-i 0^{+}}
\end{aligned}
$$

in the sense of the Sokhotski-Plemelj relation $\left(x \pm i 0^{+}\right)^{-1}=$ $\mathrm{P}(1 / x) \mp i \pi \delta(x)$ (P stands for the principal value). Assuming that the photon field is initially in the ground state and using (6) and (9) one obtains the desired modal expansion for the optical force,

$$
\mathscr{F}_{j}=\rho_{e e}(t) \Sigma_{1}+\left(1-\rho_{e e}(t)\right) \Sigma_{2},
$$

where

$$
\begin{aligned}
& \Sigma_{1}=\operatorname{Re}\left(\sum_{\omega_{n \mathbf{k}}>0} \omega_{n \mathbf{k}} \tilde{\boldsymbol{\gamma}}^{*} \cdot \partial_{j} \mathbf{F}_{n \mathbf{k}} \otimes \mathbf{F}_{n \mathbf{k}}^{*} \cdot \tilde{\gamma} \frac{1}{\omega_{n \mathbf{k}}-\omega_{0}-i 0^{+}}\right) \\
& \Sigma_{2}=\operatorname{Re}\left(\sum_{\omega_{n \mathbf{k}}>0} \omega_{n \mathbf{k}} \tilde{\boldsymbol{\gamma}} \cdot \partial_{j} \mathbf{F}_{n \mathbf{k}} \otimes \mathbf{F}_{n \mathbf{k}}^{*} \cdot \tilde{\gamma}^{*} \frac{1}{\omega_{n \mathbf{k}}+\omega_{0}-i 0^{+}}\right) .
\end{aligned}
$$

We introduced $\rho_{e e}(t)=\left\langle\hat{\sigma}_{+} \hat{\sigma}_{-}\right\rangle$, which gives the probability of the atom to be found in its excited state in a spontaneous emission process.

\section{B. Green function representation}

In what follows, it is shown that the optical force can also be expressed in terms of the Green function $\mathbf{G}$ of the system. The Green function $\mathbf{G}=\mathbf{G}\left(\mathbf{r}, \mathbf{r}^{\prime}, \omega\right)$ is a $6 \times 6$ tensor defined by Eq. (A2) of Appendix A. With the help of Eq. (A7) one may rewrite the optical force (16) as

$$
\mathscr{F}_{j}=2 \rho_{e e}(t) \operatorname{Re}\left\{\tilde{\boldsymbol{\gamma}}^{*} \cdot\left(-i \omega \partial_{j}\right) \mathbf{G}^{+}\left(\mathbf{r}_{0}, \mathbf{r}_{0}, \omega_{0}+i 0^{+}\right) \cdot \tilde{\boldsymbol{\gamma}}\right\}+2\left(1-\rho_{e e}(t)\right) \operatorname{Re}\left\{\tilde{\boldsymbol{\gamma}}^{*} \cdot\left(-i \omega \partial_{j}\right) \mathbf{G}^{-}\left(\mathbf{r}_{0}, \mathbf{r}_{0}, \omega_{0}+i 0^{+}\right) \cdot \tilde{\boldsymbol{\gamma}}\right\}
$$

where $\mathbf{G}^{ \pm}$are the positive and negative frequency parts of the Green function, and the spatial derivatives act only on the first argument (r) of the Green function. All the poles of $\mathbf{G}^{ \pm}$are in the positive and negative real frequency axes, respectively.

From Appendix A, we have $\mathbf{G}=\mathbf{G}^{+}+\mathbf{G}^{-}+$ $\frac{1}{i \omega} \mathbf{M}_{\infty}^{-1} \delta\left(\mathbf{r}-\mathbf{r}_{0}\right)$. The $\delta$-function term does not contribute to the force because it is associated with the self-field, and hence it is possible to do the replacement $\mathbf{G}^{+} \rightarrow \mathbf{G}-\mathbf{G}^{-}$in (18). This leads to Eq. (1), with the Casimir-Polder force in the ground state given by

$$
\mathscr{F}_{C, j}=2 \operatorname{Re}\left\{\left.\tilde{\gamma}^{*} \cdot\left(-i \omega \partial_{j}\right) \mathbf{G}^{-}\right|_{\omega=\omega_{0}} \cdot \tilde{\gamma}\right\} .
$$

Noting that $\mathbf{G}^{-}$is analytic for $\operatorname{Re}\{\omega\}>0$, the Cauchy theorem allows us to write the force as an integral over the imaginary frequency axis,

$$
\mathscr{F}_{C, j}=2 \operatorname{Re}\left\{\frac{1}{2 \pi} \int_{-\infty}^{\infty} d \xi \tilde{\gamma}^{*} \cdot \frac{\left(-i \omega \partial_{j} \mathbf{G}^{-}\right)_{\omega=i \xi}}{\omega_{0}-i \xi} \cdot \tilde{\gamma}\right\} .
$$

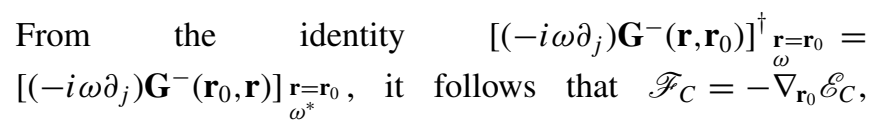

with the zero-point interaction energy given by

$$
\mathscr{E}_{C}=\frac{-1}{2 \pi} \int_{-\infty}^{\infty} d \xi \frac{1}{\omega_{0}-i \xi} \tilde{\gamma}^{*} \cdot\left(-i \omega \mathbf{G}^{-}\right)_{\omega=i \xi} \cdot \tilde{\gamma}
$$

Using again the analytic properties of $\mathbf{G}^{-}$, we see that $0=$ $\frac{1}{2 \pi} \int_{-\infty}^{\infty} d \xi \frac{1}{\omega_{0}+i \xi}\left(-i \omega \mathbf{G}^{-}\right)_{\omega=i \xi}$. Thus, introducing the tensor $\tilde{\alpha}=\left(\frac{1}{\omega_{0}-\omega} \tilde{\gamma} \tilde{\gamma}^{*}+\frac{1}{\omega_{0}+\omega} \tilde{\gamma}^{*} \tilde{\gamma}\right)$, which corresponds to a normalized polarizability of the two-level atom, it is possible to write

$$
\mathscr{E}_{C}=\frac{-1}{2 \pi} \int_{-\infty}^{\infty} d \xi \operatorname{tr}\left(\tilde{\alpha}(i \xi) \cdot\left(-i \omega \mathbf{G}^{-}\right)_{\omega=i \xi}\right)
$$

Noting finally that $\left(-i \omega \overline{\mathbf{G}}^{-}\left(\mathbf{r}_{0}, \mathbf{r}_{0}, \omega\right)\right)_{\omega=i \xi}=$ $\left[\left(-i \omega \overline{\mathbf{G}}^{+}\left(\mathbf{r}_{0}, \mathbf{r}_{0}, \omega\right)\right)_{\omega=i \xi}\right]^{*}$ and that $\tilde{\alpha}(i \xi)=\tilde{\alpha}^{*}(i \xi)$, and taking into account that the integral is necessarily real-valued, we see that the interaction Casimir energy may be calculated using (22) with $\mathbf{G}^{+}$in the place of $\mathbf{G}^{-}$. This result also implies that we can replace $\mathbf{G}^{-}$by one-half of the full Green function $\mathbf{G} / 2$ in (22), and this final observation yields the desired 
Eq. (3), which may be written as

$$
\begin{aligned}
\mathscr{E}_{C}= & -\frac{1}{4 \pi} \int_{-\infty}^{\infty} d \xi\left(\frac{1}{\omega_{0}-i \xi} \tilde{\gamma}^{*} \cdot(-i \omega \mathbf{G})_{\omega=i \xi} \cdot \tilde{\gamma}\right. \\
& \left.+\frac{1}{\omega_{0}+i \xi} \tilde{\gamma} \cdot(-i \omega \mathbf{G})_{\omega=i \xi} \cdot \tilde{\gamma}^{*}\right) .
\end{aligned}
$$

This completes the proof of Eqs. (1)-(3). Even though the derivation assumes negligible material loss, the final result is given in terms of the Green function, and thus it can be readily extended to lossy material systems simply by using the Green function for lossy systems in the same expression. As previously mentioned, only the scattering part of the Green function needs to be considered in the force calculation, because by symmetry the self-field (i.e., the part of the Green function associated with the radiation of an electric dipole in a vacuum) does not contribute to the force.

Equation (1) generalizes (in the low-temperature limit) the theory of Refs. [12,15] (which applies only to isotropic dielectrics) to arbitrary bianisotropic (reciprocal or nonreciprocal) material platforms. Note that different from Refs. [12,15] our theory neglects atomic level shifts and broadenings. It is worth pointing out that for reciprocal systems the integral in Eq. (3) can be reduced to the positive imaginary axis, but for general nonreciprocal systems the integration must be over the entire imaginary axis.

\section{Lateral force for stratified systems}

So far the analysis is completely general (under the electric dipole approximation), and applies to a generic system with no particular symmetries. Next, we focus on structures invariant to translations along the coordinates $\alpha=x, y$, and discuss some properties of the optical lateral force in such systems.

Clearly, for a structure invariant to translations along $\alpha=$ $x, y$ the force component $\mathscr{F}_{C, \alpha}$ vanishes. Furthermore, in the limit of no material loss we find from (17) with the SokhotskiPlemelj relation, and using the fact that the modes are Bloch waves, that

$$
\begin{aligned}
& \mathscr{F}_{\alpha}=\rho_{e e}(t) \\
& \times \operatorname{Re}\left(i \pi \sum_{\omega_{n \mathbf{k}}>0} \omega_{n \mathbf{k}} \tilde{\boldsymbol{\gamma}}^{*} \cdot \partial_{\alpha} \mathbf{F}_{n \mathbf{k}} \otimes \mathbf{F}_{n \mathbf{k}}^{*} \cdot \tilde{\boldsymbol{\gamma}} \delta\left(\omega_{n \mathbf{k}}-\omega_{0}\right)\right) .
\end{aligned}
$$

In general, for lossy materials, the modal expansion does not apply and one needs to use Eq. (2). As mentioned, the force only depends on the "electric part" of the Green function $\mathbf{G}_{\mathrm{EE}}$. The vector $\mathbf{E}=-i \omega \mathbf{G}_{\mathrm{EE}} \cdot \boldsymbol{\gamma}$ corresponds to the frequency domain electric field radiated by a classical dipole with electric dipole moment $\boldsymbol{\gamma}$. The exact lateral force can be written in terms of this electric field as follows (only the scattering part of the field needs to be considered),

$$
\mathscr{F}_{\alpha}=2 \rho_{e e}(t) \operatorname{Re}\left\{\boldsymbol{\gamma}^{*} \cdot \partial_{\alpha} \mathbf{E}\left(\mathbf{r}_{0}\right)\right\} .
$$

The application of these formulas is illustrated in [39].

\section{Multilevel atom}

The formalism developed in the previous sections can be readily generalized to a multilevel atom described by the
Hamiltonian $\hat{H}_{\text {at }}=\sum_{n} E_{n}|n\rangle\langle n|$, with $E_{n}$ the energy level of the $n$th state. It is supposed that the dipole moment matrix $\boldsymbol{\gamma}_{m n}=\langle m|\hat{\mathbf{p}}| n\rangle$ has no diagonal elements. Thus, it is possible to write the dipole moment operator as (for simplicity it is assumed there are no degenerate levels)

$$
\hat{\mathbf{p}}=\sum_{E_{m}<E_{n}}\left(\boldsymbol{\gamma}_{m n}^{*}|n\rangle\left\langle m\left|+\boldsymbol{\gamma}_{m n}\right| m\right\rangle\langle n|\right),
$$

which may be understood as a combination of multiple twolevel systems. Because Maxwell's equations are linear, Eq. (15) can be readily generalized to a multilevel system by including the contribution of each "two-level" term. Then, substituting this result into (9) one sees that since the Heisenberg equations preserve the orthogonality relations, $\langle m(t) \mid n(t)\rangle=\delta_{m, n}$, each "two-level" component of the atom Hamiltonian contributes independently to the force. Note that we assume that in the initial state the electromagnetic field has no quanta. This result proves that the optical force is a superposition of the individual "two-level" contributions:

$$
\mathscr{F}_{i}(t)=\sum_{E_{m}<E_{n}} \rho_{n n}(t) \mathscr{F}_{R, i}^{m n}+\left(\rho_{m m}(t)-\rho_{n n}(t)\right) \mathscr{F}_{C, i}^{m n} .
$$

Here, $\rho_{n n}(t)$ is the probability of finding the atom in the $n$th state at time $t$, and $\mathscr{F}_{R, i}^{m n}$ and $\mathscr{F}_{C, i}^{m n}$ are calculated using Eqs. (2) and (3) with $\boldsymbol{\gamma}_{m n}$ in the place of $\boldsymbol{\gamma}$ and $\omega_{0, m n}=\left(E_{n}-E_{m}\right) / \hbar$ in the place of $\omega_{0}$.

\section{TOPOLOGICAL MATERIAL HALF-SPACE}

In the rest of the article, we focus on a $z$-stratified structure formed by a topological material half-space $(z<0)$ and a free-space half-space $(z>0)$ (Fig. 1). The atom is located a distance $d$ above the topological material. It is assumed that the material only has a nontrivial electric response, so that $\mathbf{M}=\left(\begin{array}{cc}\varepsilon(\mathbf{r}, \omega) & 0 \\ 0 & \mu_{0} \mathrm{I}\end{array}\right)$. Furthermore, we suppose that the material response is gyrotropic with dielectric function,

$$
\boldsymbol{\varepsilon}=\varepsilon_{0}\left(\varepsilon_{t} \mathbf{I}_{t}+\varepsilon_{a} \hat{\mathbf{y}} \hat{\mathbf{y}}+i \varepsilon_{g} \hat{\mathbf{y}} \times \mathbf{I}\right),
$$

where $\mathbf{I}_{t}=\mathbf{I}-\hat{\mathbf{y}} \hat{\mathbf{y}}$ and $\varepsilon_{g}$ determines the strength of the nonreciprocal response.

In Appendix B, we derive an explicit formula for the "electric part" of the Green function $\mathbf{G}_{\mathrm{EE}}$ in the region $z>$ 0 and $z^{\prime}>0$. The Green function has the decomposition $\mathbf{G}_{\mathrm{EE}}=\mathbf{G}_{\mathrm{EE}, 0}+\mathbf{G}_{\mathrm{EE}, \mathrm{s}}$, with $\mathbf{G}_{\mathrm{EE}, 0}$ the free-space Green function (associated with the self-field) given by $\left(-i \omega \varepsilon_{0}\right) \mathbf{G}_{\mathrm{EE}, 0}=$

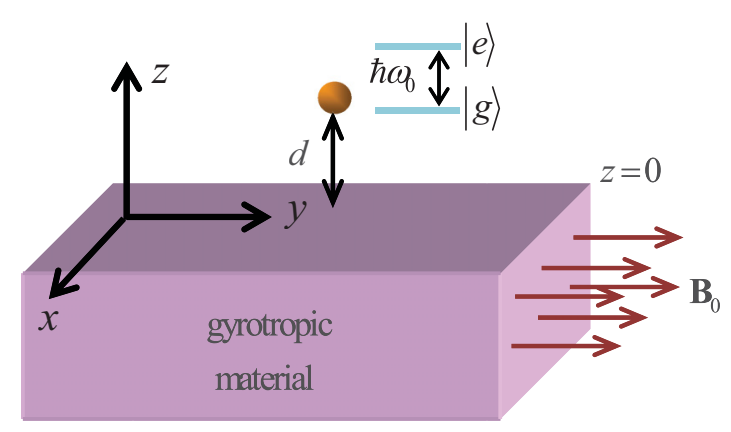

FIG. 1. A two-level system is at a distance $d$ above a gyrotropic material. 
$\left(\nabla \nabla+k_{0}^{2} \mathrm{I}\right) \Phi_{0}$ where $\Phi_{0}=e^{i k_{0} r} / 4 \pi r$. The scattering part of the Green function $\mathbf{G}_{\mathrm{EE}, \mathrm{s}}$ is given by a Sommerfeld-type integral,

$$
\begin{aligned}
& \left(-i \omega \varepsilon_{0}\right) \mathbf{G}_{\mathrm{EE}, s}\left(\mathbf{r}, \mathbf{r}^{\prime}\right) \\
& =\frac{1}{(2 \pi)^{2}} \iint d k_{x} d k_{y} \frac{e^{-\gamma_{0}\left(z+z^{\prime}\right)}}{2 \gamma_{0}} e^{i \mathbf{k}_{\| \cdot\left(\mathbf{r}-\mathbf{r}^{\prime}\right)}} \mathbf{C}\left(\omega, \mathbf{k}_{\|}\right),
\end{aligned}
$$

where $\quad \mathbf{k}_{\|}=k_{x} \hat{\mathbf{x}}+k_{y} \hat{\mathbf{y}}, \quad \gamma_{0}=\sqrt{k_{\|}^{2}-k_{0}^{2}}, \quad k_{0}=\omega / c, \quad$ and $\mathbf{C}\left(\omega, \mathbf{k}_{\|}\right)$is the tensor defined by Eq. (B3), which is written in terms of the reflection matrix for the gyrotropic material half-space. To the best of our knowledge, this is the first time that the Green function of a gyrotropic half-space is determined explicitly as a Sommerfeld integral.

By substituting (29) into (1)-(3) one obtains the exact solution for the optical force within the Markov approximation for a transition between an excited state and the ground. For example, the resonant component of the force can be written as

$$
\mathscr{F}_{R, i}=2 \operatorname{Re}\left\{\left.\gamma^{*} \cdot\left(-i \omega \partial_{i} \mathbf{G}_{\mathrm{EE}}\left(\mathbf{r}, \mathbf{r}_{0} ; \omega\right)\right)\right|_{\substack{\omega=\omega_{0}+i 0^{+} \\ \mathbf{r}=\mathbf{r}_{0}}} \cdot \gamma\right\},
$$

with $\mathbf{r}_{0}=(0,0, d)$

For a lossy magnetized plasma with bias magnetic field along the $+y$ axis the permittivity elements are [50]

$$
\begin{aligned}
& \varepsilon_{t}=1-\frac{\omega_{p}^{2}(1+i \Gamma / \omega)}{(\omega+i \Gamma)^{2}-\omega_{c}^{2}}, \\
& \varepsilon_{a}=1-\frac{\omega_{p}^{2}}{\omega(\omega+i \Gamma)}, \quad \varepsilon_{g}=\frac{1}{\omega} \frac{\omega_{c} \omega_{p}^{2}}{\omega_{c}^{2}-(\omega+i \Gamma)^{2}} .
\end{aligned}
$$

Here, $\omega_{p}$ is the plasma frequency, $\Gamma$ is the collision rate associated with damping, $\omega_{c}=-q B_{0} / m$ is the cyclotron frequency, $q=-e$ is the electron charge, $m$ is the effective electron mass, and $B_{0}$ is the static bias. The cyclotron frequency is either positive or negative depending if $B_{0}$ is oriented along the $+y$ or $-y$ direction, respectively. Narrow gap semiconductors such as InSb have a response analogous to (31) [51,52].

It has been recently shown that electromagnetic continua with no intrinsic periodicity but with broken time-reversal symmetry, e.g., the biased plasma described by (31), can be understood as topological materials. In particular, such materials enable the propagation of unidirectional, topologically protected, and scattering-immune edge states [34-36,47].
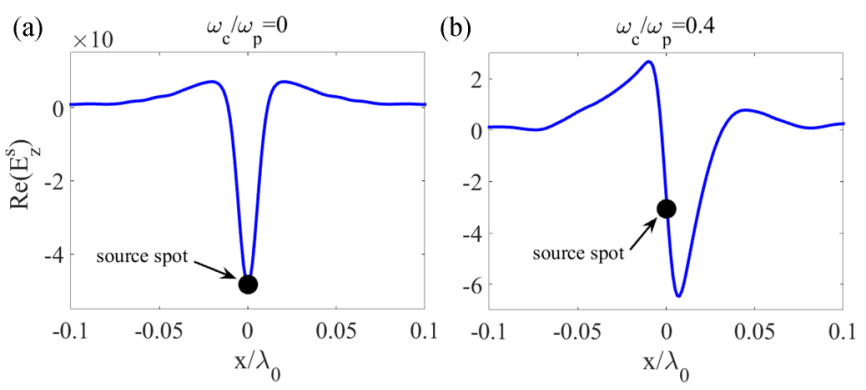

FIG. 2. Real part of the scattered electric field (in arbitrary units) near the source for the unbiased plasma (a) and the biased plasma (b). The oscillation frequency is $\omega / \omega_{p}=0.7$ and $\lambda_{0}=2 \pi c / \omega$. The source (black dot) is located at $d=0.05 c / \omega_{p}$ above the magnetized plasma.
From (2) and (25) it is seen that the lateral force is determined by the slope of the Green function and electric field at the atom position [39]. To illustrate that the slope is nonzero in the nonreciprocal case, we consider that the dipole is polarized along the vertical $(z)$ direction $\left(\mathbf{E}=-i \omega \mathbf{G}_{\mathrm{EE}} \cdot \gamma \hat{\mathbf{z}}\right)$. As shown in Fig. 2(a), for an unbiased plasma (when $\omega_{c}=0$ and the dielectric function reduces to the Drude dispersion scalar model), $\partial_{x} E_{z}=0$ at the source point, and therefore $\mathscr{F}_{x}=0$. However, in the presence of a magnetic bias the field at the atom position has nonzero slope [Fig. 2(b)], and hence $\mathscr{F}_{x} \neq 0$. Note that Fig. 2 shows only the scattered part of the field at the source point.

\section{QUASISTATIC SOLUTION}

To have some physical insight into the mechanisms that determine the optical force, next we obtain an explicit expression for the force under the assumption that $d \ll 2 \pi c / \omega_{p}$ and $d \ll$ $2 \pi c / \omega_{0}$ (quasistatic limit) and that the material absorption is negligible.

\section{A. Surface plasmon polaritons}

When the atom is in close vicinity of the topological material, the light-matter interactions are expected to be mainly determined by the surface plasmon polaritons (SPPs). In the following, we derive the dispersion of the SPP resonances (i.e., SPPs with short wavelengths and wave vector $\mathbf{k}_{\|} \rightarrow \infty$ ) based on the quasistatic approximation. The propagation of SPPs has been widely discussed in the literature mainly when the direction of propagation is perpendicular to the bias field (e.g., $[34,53,54])$, but it seems that for oblique directions the problem was not systematically studied so far.

It is well known that SPPs with short wavelengths have an electrostatic nature. Thus, we look for guided modes of the form $\mathbf{F}_{n \mathbf{k}} \approx\left[\mathbf{E}_{n \mathbf{k}} \mathbf{0}\right]^{\mathrm{T}} \approx\left[-\nabla \phi_{\mathbf{k}} \mathbf{0}\right]^{\mathrm{T}}$. The magnetic field is assumed negligible and the electric field is written in terms of an electric potential $\left(\phi_{\mathbf{k}}\right)$ that satisfies $\nabla \cdot\left(\varepsilon \cdot \nabla \phi_{\mathbf{k}}\right)=0$. The solutions of this quasistatic equation are of the form,

$$
\phi_{\mathbf{k}}=\frac{A_{\mathbf{k}_{\|}}}{\sqrt{S}} e^{i \mathbf{k}_{\| \cdot} \cdot \mathbf{r}}\left\{\begin{array}{ll}
e^{-k_{\|} z}, & z>0 \\
e^{+\tilde{k}_{\|} z}, & z<0
\end{array},\right.
$$

where $\mathbf{k}_{\|}=k_{x} \hat{\mathbf{x}}+k_{y} \hat{\mathbf{y}}$ is the wave vector of the SPPs, $A_{\mathbf{k}_{\|}}$is a normalization parameter, $\tilde{k}_{\|}=\sqrt{k_{x}^{2}+\left(\varepsilon_{a} / \varepsilon_{t}\right) k_{y}^{2}}$, and $S$ is the area of the slab.

Imposing that the normal component of the electric displacement is continuous at the interface, i.e., that $\hat{\mathbf{z}} \cdot \varepsilon \cdot \nabla \phi_{\mathbf{k}}$ is continuous at $z=0$, we obtain the condition for the SPP resonance,

$$
-k_{\|}=k_{x} \varepsilon_{g}(\omega)+\tilde{k}_{\|} \varepsilon_{t}(\omega) .
$$

For the dispersive model (31), the solution of (33) yields a single branch of modes $\omega_{\mathbf{k}}$, which depends only on the angle $\theta$ of the wave vector with respect to the $x$ axis, not on its magnitude,

$$
\omega_{\mathbf{k}}=\omega_{\theta}=\frac{\omega_{c}}{2} \cos (\theta)+\sqrt{\frac{\omega_{p}^{2}}{2}+\frac{\omega_{c}^{2}}{4}\left(1+\sin ^{2}(\theta)\right)} .
$$


For $\omega_{c}>0$, one has $\omega_{-}<\omega_{\mathbf{k}}<\omega_{+}$, with

$$
\begin{aligned}
& \omega_{+} \equiv \omega_{k_{x}>0, k_{y}=0}=\frac{1}{2}\left(\omega_{c}+\sqrt{2 \omega_{p}^{2}+\omega_{c}^{2}}\right), \\
& \omega_{-} \equiv \omega_{k_{x}<0, k_{y}=0}=\frac{1}{2}\left(-\omega_{c}+\sqrt{2 \omega_{p}^{2}+\omega_{c}^{2}}\right) .
\end{aligned}
$$

To have some insight into the physical meaning of the SPP resonance, we numerically calculated the exact dispersion of the surface plasmons using the formalism presented in Appendix C. Figure 3 depicts $\omega_{\mathbf{k}}^{\text {SPP }}$ (the exact SPP dispersion) versus $k_{\|}$along different directions $\theta$ of the wave vector. In each panel, the dashed horizontal line marks the SPP resonance for which $\omega_{\mathbf{k}_{\|}}^{\mathrm{SPP}} \rightarrow \omega_{\theta}$ with $\omega_{\theta}$ given by (34). Thus, the quasistatic analytic solution determines the SPPs with very short wavelengths $\left(\mathbf{k}_{\|} \rightarrow \infty\right)$.

In the limit of a vanishing bias field, $B_{0} \rightarrow 0$, the permittivity has a standard Drude dispersion. In such a case, the SPP resonance becomes angle independent,

$$
\begin{gathered}
\lim _{\omega_{c} \rightarrow 0} \omega_{\mathbf{k}}=\frac{\omega_{p}}{\sqrt{2}}, \quad \text { (36) components) may be written as } \\
\mathscr{F}=\rho_{e e}(t) \operatorname{Re}\left(\sum_{\omega_{\mathbf{k}}>0} \omega_{\mathbf{k}} \frac{\left|A_{\mathbf{k}_{\|}}\right|^{2}}{S} e^{-2 k_{\|} d}\left(i \mathbf{k}_{\|}-k_{\|} \hat{\mathbf{z}}\right) \boldsymbol{\gamma}^{*} \cdot\left(i \mathbf{k}_{\|}-k_{\|} \hat{\mathbf{z}}\right)\left(-i \mathbf{k}_{\|}-k_{\|} \hat{\mathbf{z}}\right) \cdot \boldsymbol{\gamma} \frac{1}{\omega_{\mathbf{k}}-\omega_{0}-i 0^{+}}\right) \\
+\left(1-\rho_{e e}(t)\right) \operatorname{Re}\left(\sum_{\omega_{\mathbf{k}}>0} \omega_{\mathbf{k}} \frac{\left|A_{\mathbf{k}_{\|}}\right|^{2}}{S} e^{-2 k_{\|} d}\left(i \mathbf{k}_{\|}-k_{\|} \hat{\mathbf{z}}\right) \boldsymbol{\gamma} \cdot\left(i \mathbf{k}_{\|}-k_{\|} \hat{\mathbf{z}}\right)\left(-i \mathbf{k}_{\|}-k_{\|} \hat{\mathbf{z}}\right) \cdot \boldsymbol{\gamma}^{*} \frac{1}{\omega_{\mathbf{k}}+\omega_{0}-i 0^{+}}\right) .
\end{gathered}
$$

To proceed, we use $\frac{1}{S} \sum_{\omega_{\mathbf{k}}>0} \rightarrow \frac{1}{(2 \pi)^{2}} \iint d k_{x} d k_{y}$ to transform the summation over the discrete modes into an integral. Moreover, using polar coordinates $\mathbf{k}_{\|}=k_{\|}(\cos \theta, \sin \theta, 0)$, and noting that $\omega_{\mathbf{k}}=\omega_{\theta}$, it is possible to write

$$
\begin{aligned}
\mathscr{F}= & \rho_{e e}(t) \frac{|\gamma|^{2}}{\varepsilon_{0}} \operatorname{Re}\left\{\frac{1}{(2 \pi)^{2}} \int_{0}^{2 \pi} d \theta \int_{0}^{\infty} d k_{\|} k_{\|}^{2} \omega_{\theta} a_{\theta} e^{-2 k_{\|} d} \Gamma_{+, \theta} \frac{1}{\omega_{\theta}-\omega_{0}-i 0^{+}}\left(i \mathbf{k}_{\|}-k_{\|} \hat{\mathbf{z}}\right)\right\} \\
& +\left(1-\rho_{e e}(t)\right) \frac{|\gamma|^{2}}{\varepsilon_{0}} \operatorname{Re}\left\{\frac{1}{(2 \pi)^{2}} \int_{0}^{2 \pi} d \theta \int_{0}^{\infty} d k_{\|} k_{\|}^{2} \omega_{\theta} a_{\theta} e^{-2 k_{\|} d} \Gamma_{-, \theta} \frac{1}{\omega_{\theta}+\omega_{0}-i 0^{+}}\left(i \mathbf{k}_{\|}-k_{\|} \hat{\mathbf{z}}\right)\right\},
\end{aligned}
$$

where we introduced

$$
\begin{aligned}
& \Gamma_{+, \theta}=\frac{1}{|\gamma|^{2} k_{\|}^{2}}\left|\left(-i \mathbf{k}_{\|}-k_{\|} \hat{\mathbf{z}}\right) \cdot \boldsymbol{\gamma}\right|^{2}, \\
& \Gamma_{-, \theta}=\frac{1}{|\gamma|^{2} k_{\|}^{2}}\left|\left(-i \mathbf{k}_{\|}-k_{\|} \hat{\mathbf{z}}\right) \cdot \boldsymbol{\gamma}^{*}\right|^{2},
\end{aligned}
$$

and $a_{\theta} \equiv\left|A_{\mathbf{k}_{\|}}\right|^{2} \varepsilon_{0} k_{\|}$, which are functions only of $\theta$, not of $k_{\|}$. The integrals over $k_{\|}$can be explicitly evaluated using $\int_{0}^{\infty} e^{-2 k_{\|} d} k_{\|}^{3} d k_{\|}=\frac{3}{8} \frac{1}{d^{4}}$.

For the lateral force, only the two poles $\theta= \pm \theta_{0}$ for which the plasmon frequency matches the transition frequency of the two-level atom $\left(\omega_{ \pm \theta_{0}}=\omega_{0}\right)$ contribute to the integral. In this case, we find that

$$
\begin{aligned}
& \frac{\mathscr{F}_{x}}{\mathscr{F}_{0}}=-\left.\rho_{e e}(t) \frac{\omega_{\theta} a_{\theta} \cos \theta}{\left|\partial_{\theta} \omega_{\theta}\right|}\right|_{\theta=\theta_{0}} \frac{1}{2}\left(\Gamma_{+, \theta_{0}}+\Gamma_{+,-\theta_{0}}\right), \\
& \frac{\mathscr{F}_{y}}{\mathscr{F}_{0}}=-\left.\rho_{e e}(t) \frac{\omega_{\theta} a_{\theta} \sin \theta}{\left|\partial_{\theta} \omega_{\theta}\right|}\right|_{\theta=\theta_{0}} \frac{1}{2}\left(\Gamma_{+, \theta_{0}}-\Gamma_{+,-\theta_{0}}\right) .
\end{aligned}
$$

with

$$
\mathscr{F}_{0}=\frac{3|\gamma|^{2}}{16 \pi d^{4} \varepsilon_{0}},
$$

a normalizing parameter with unities of force $(\mathrm{N})$. Thus, in the quasistatic approximation, the recoil force decays as $1 / d^{4}$ with respect to the distance to the interface [39]. Note that for a $z$-directed dipole $\gamma=\gamma \hat{\mathbf{z}}$, we have $\Gamma_{+, \pm \theta}=1$. As discussed in detail in [39], since $a_{\theta}>0$ and $\Gamma_{+, \pm \theta} \geqslant 0$ the sign of the force component $\mathscr{F}_{x}$ (lateral force perpendicular to the bias magnetic field) is independent of the dipole polarization and orientation. Furthermore, the sign of $\mathscr{F}_{x}$ can be tuned with the 

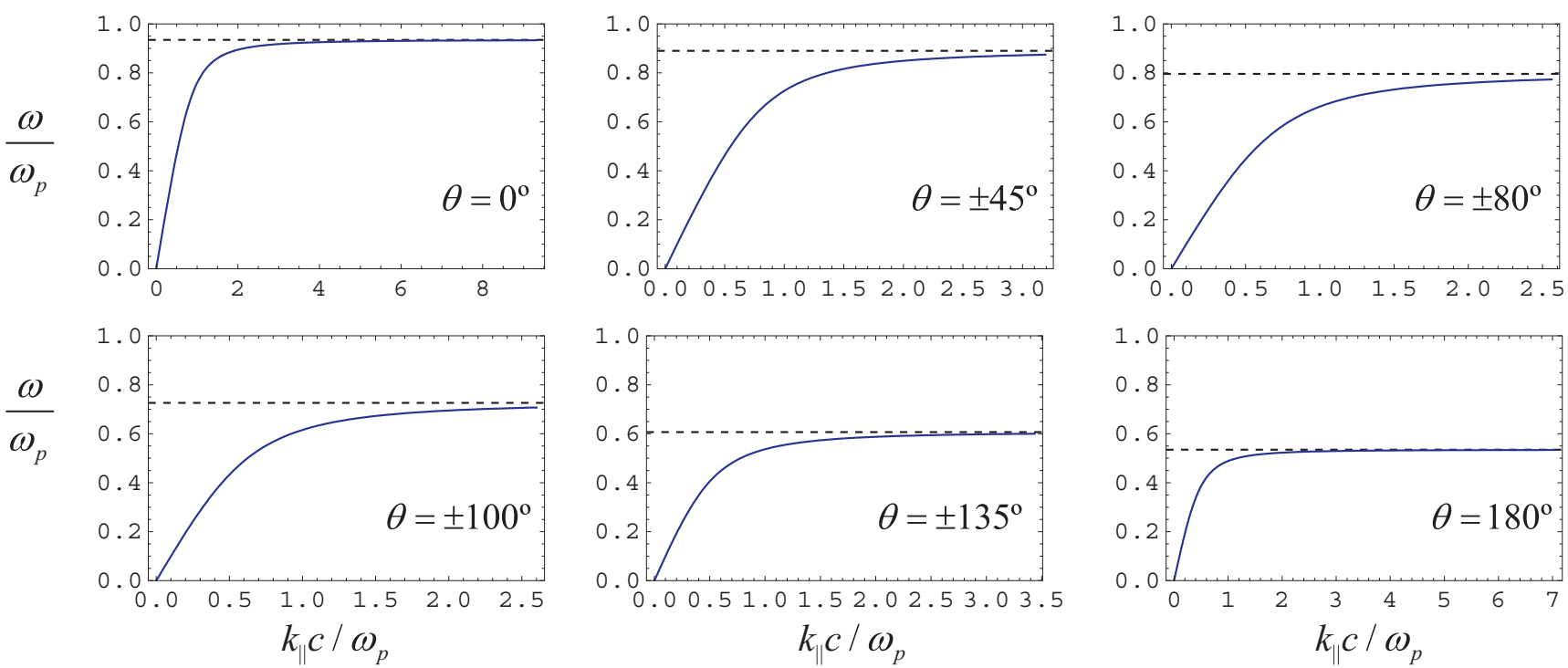

FIG. 3. Exact dispersion of the surface plasmons for different angles $\theta$ of the wave vector. The dashed horizontal lines mark the value of the quasistatic SPP resonance $\left(\omega_{\theta}\right)$ determined by (34). The cyclotron frequency is $\omega_{c}=0.4 \omega_{p}$.

applied bias field. In contrast, the sign of $\mathscr{F}_{y}$ depends on the polarization state. It is highlighted that the equation $\omega_{ \pm \theta_{0}}=\omega_{0}$ has a solution only if $\omega_{-} \leqslant \omega_{0} \leqslant \omega_{+}$. When $\omega_{0}$ lies outside the frequency range of the SPP resonances there are no poles, and the quasistatic approximation predicts a vanishing lateral force. Indeed, plasmons with long wavelengths interact weakly with the atom. Consistent with this, it is shown in [39] that the exact lateral force quickly approaches zero when $\omega_{0}<\omega_{-}$or $\omega_{0}>\omega_{+}$.

Equation (41) reveals that the lateral force is mainly determined by the plasmons that propagate with wave vector directed along either $\theta=\theta_{0}$ or $\theta=-\theta_{0}$. As further discussed in [39], this implies that the momentum transfer is determined by the canonical (Minkowski) momentum of light, parallel to the wave vector, rather than by the kinetic (Abraham) momentum, parallel to the Poynting vector (or equivalently, to the group velocity).

The vertical component of the force is

$$
\begin{aligned}
\frac{\mathscr{F}_{z}}{\mathscr{F}_{0}}= & -\rho_{e e}(t) \operatorname{Re}\left\{\frac{1}{2 \pi} \int_{0}^{2 \pi} d \theta a_{\theta} \omega_{\theta} \Gamma_{+, \theta} \frac{1}{\omega_{\theta}-\omega_{0}-i 0^{+}}\right\} \\
& -\left(1-\rho_{e e}(t)\right) \operatorname{Re}\left\{\frac{1}{2 \pi} \int_{0}^{2 \pi} d \theta a_{\theta} \omega_{\theta} \Gamma_{-, \theta} \frac{1}{\omega_{\theta}+\omega_{0}}\right\}
\end{aligned}
$$

In the steady-state limit, the only contribution to the normal force is from the second integral, giving the Casimir-Polder force $\left(\mathscr{F}_{C}=\mathscr{F}_{z, t \rightarrow \infty}\right)$ due to the vacuum fluctuations at zero temperature,

$$
\frac{\mathscr{F}_{C}}{\mathscr{F}_{0}}=-\frac{1}{2 \pi} \int_{0}^{2 \pi} d \theta a_{\theta} \omega_{\theta} \Gamma_{-, \theta} \frac{1}{\omega_{0}+\omega_{\theta}} .
$$

The Casimir-Polder force is clearly attractive $\left(\mathscr{F}_{C}<0\right)$. The sign of the dynamic normal force [Eq. (43)] may be either positive or negative.

\section{Limit of weak bias field}

It is interesting to further analyze the quasistatic solution in the limit of a weak bias field $\omega_{c} \rightarrow 0$. Without loss of generality, we suppose that the atom dipole moment is directed along $z$, so that $\Gamma_{+, \pm \theta_{0}}=1$. It can be shown that for a weak bias magnetic field $\omega_{\theta} \approx \omega_{\text {spp }}+\frac{\omega_{c}}{2} \cos \theta$ and $a_{\theta} \approx 1 / 2$, so that $\left|\partial_{\theta} \omega_{\theta}\right|_{\theta=\theta_{0}} \approx\left|\omega_{c} \sin \theta_{0}\right| / 2$ with $\omega_{\text {spp }}=\omega_{p} / \sqrt{2}$. Thus, the solution of $\omega_{ \pm \theta_{0}}=\omega_{0}$ is such that $\cos \theta_{0}=2\left(\omega_{0}-\omega_{\text {spp }}\right) / \omega_{c}$. Therefore, for a weak bias and $\left|\omega_{0}-\omega_{\text {spp }}\right|<\left|\omega_{c}\right| / 2$ the nonzero component of the lateral force (41) reduces to

$$
\frac{\mathscr{F}_{x}}{\mathscr{F}_{0}}=-\rho_{e e}(t) \frac{\omega_{\text {spp }}}{\omega_{c}} \frac{\omega_{0}-\omega_{\text {spp }}}{\sqrt{\left(\omega_{c} / 2\right)^{2}-\left(\omega_{0}-\omega_{\text {spp }}\right)^{2}}} .
$$

Remarkably, as further discussed in [39], the quasistatic theory predicts that the lateral force diverges in the $\omega_{c} \rightarrow 0$ limit and for $\left|\omega_{0}-\omega_{\text {spp }}\right|=\left|\omega_{c}\right| / 2$, i.e., when $\omega_{0}=\omega_{+}$or $\omega_{0}=\omega_{-}$. The lateral force vanishes when $\left|\omega_{0}-\omega_{\text {spp }}\right|>\left|\omega_{c}\right| / 2$.

Furthermore, for a weak bias and $\left|\omega_{0}-\omega_{\text {spp }}\right|<\left|\omega_{c}\right| / 2$ the normal force (43) is simply $\mathscr{F}_{z}=\left(1-\rho_{e e}(t)\right) \mathscr{F}_{C}$, with

$$
\frac{\mathscr{F}_{C}}{\mathscr{F}_{0}}=-\frac{1}{2} \frac{\omega_{\text {spp }}}{\omega_{\text {spp }}+\omega_{0}}
$$

the Casimir-Polder force when the atom is in the ground state. In contrast, for $\left|\omega_{0}-\omega_{\mathrm{spp}}\right|>\left|\omega_{c}\right| / 2$ the normal force gains an additional resonant component:

$$
\begin{aligned}
\frac{\mathscr{F}_{z}}{\mathscr{F}_{0}}= & -\rho_{e e}(t) \frac{\omega_{\mathrm{spp}}}{2} \frac{\operatorname{sgn}\left(\omega_{\mathrm{spp}}-\omega_{0}\right)}{\sqrt{\left(\omega_{0}-\omega_{\mathrm{spp}}\right)^{2}-\left(\omega_{c} / 2\right)^{2}}} \\
& -\left(1-\rho_{e e}(t)\right) \frac{1}{2} \frac{\omega_{\mathrm{spp}}}{\omega_{\mathrm{spp}}+\omega_{0}} .
\end{aligned}
$$

Hence, the quasistatic theory also predicts that the normal force diverges when $\omega_{0}=\omega_{+}$or $\omega_{0}=\omega_{-}$. In contrast, the force component $\mathscr{F}_{C}$ has no resonances. It will be shown in Sec. V that the force calculated with the "exact" Green function is 

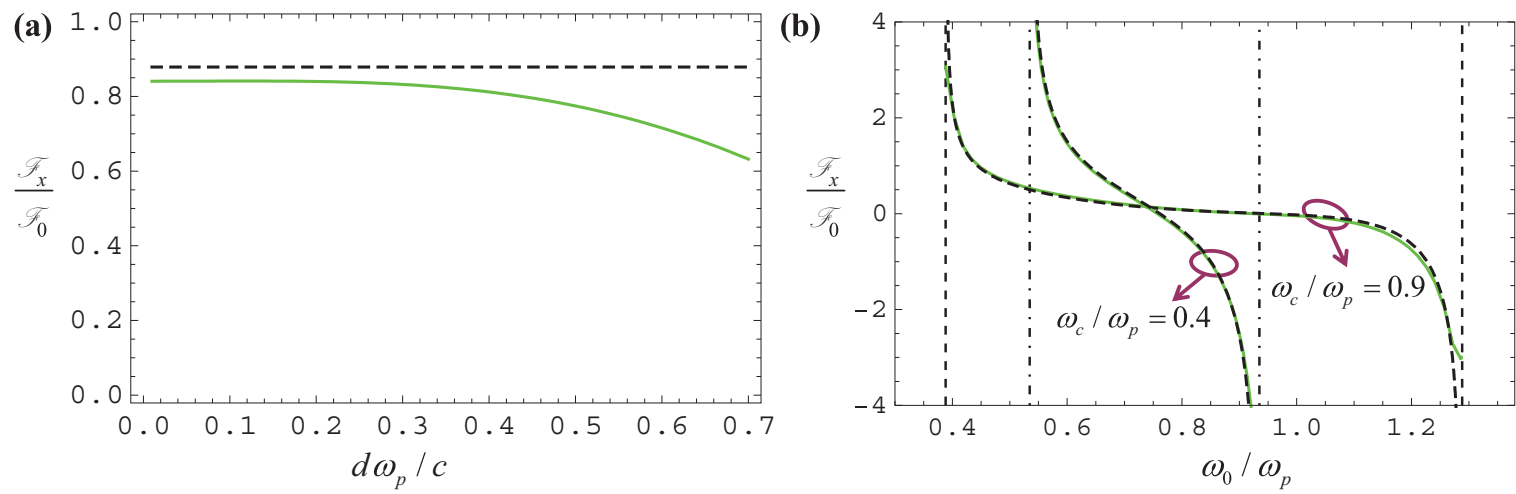

FIG. 4. Comparison of the quasistatic (dashed black lines) and exact (solid green lines) solutions for the lateral force for an excited atom $\left(\rho_{e e}=1\right)$. In the "exact" calculation the plasma collision frequency is taken equal to $\Gamma=0.015 \omega_{p}$. (a) Lateral force as a function of the atom-interface distance $d$ for a biased plasma with $\omega_{c} / \omega_{p}=0.4$ and an atom with $\omega_{0} / \omega_{p}=0.65$. (b) Lateral force as a function of $\omega_{0}$ for the distance $d=0.01 c / \omega_{p}$. The plot only shows the frequency range $\omega_{-}<\omega_{0}<\omega_{+}$, which for each case is delimited by the vertical grid lines.

finite when material loss and time retardation are taken into account.

If the atom is prepared in an excited state, $\rho_{e e}(t=0)=1$, it can be seen from (45) that the sign of the lateral force $\mathscr{F}_{x, t=0}$ can be controlled either by changing $\omega_{c}$ or $\omega_{0}$. In contrast, from (47), the sign of the normal force $\mathscr{F}_{z, t=0}$ only depends on $\omega_{0}$, and hence cannot be dynamically tuned by flipping the bias field.

\section{Influence of the atom polarization}

The atom polarization influences the strength of the Casimir-Polder force through the non-negative coefficients $\Gamma_{ \pm, \theta}$. In particular, the lateral force $\mathscr{F}_{x}$ depends on the sum $\Gamma_{+, \theta_{0}}+\Gamma_{+,-\theta_{0}}$.

From (40) it is simple to check that $\Gamma_{+, \theta_{0}}=$ $\frac{1}{|\boldsymbol{\gamma}|^{2}}\left|\left(i \cos \theta_{0} \hat{\mathbf{x}}+i \sin \theta_{0} \hat{\mathbf{y}}+\hat{\mathbf{z}}\right) \cdot \boldsymbol{\gamma}\right|^{2}$, and thereby it is evident that $0 \leqslant \Gamma_{+, \theta_{0}} \leqslant 2$. The maximum $\left(\Gamma_{+, \theta_{0}}=2\right)$ is achieved for a polarization state such that $\boldsymbol{\gamma} \sim-i \cos \theta_{0} \hat{\mathbf{x}}-i \sin \theta_{0} \hat{\mathbf{y}}+\hat{\mathbf{z}}$.

The minimum $\Gamma_{+, \theta_{0}}=0$ is attained when $\boldsymbol{\gamma}$ belongs to a two-dimensional complex vector space generated by the complex vectors $\mathbf{v}_{1, \theta_{0}}=i \cos \theta_{0} \hat{\mathbf{x}}+i \sin \theta_{0} \hat{\mathbf{y}}+\hat{\mathbf{z}}$ and $\mathbf{v}_{2, \theta_{0}}=$ $-\sin \theta_{0} \hat{\mathbf{x}}+\cos \theta_{0} \hat{\mathbf{y}}$. Similarly, the function $\Gamma_{+,-\theta_{0}}$ vanishes when that atom polarization lies in the two-dimensional complex vector space generated by the vectors $\mathbf{v}_{1,-\theta_{0}}$ and $\mathbf{v}_{2,-\theta_{0}}$. Thus, it follows that $\Gamma_{+, \theta_{0}}+\Gamma_{+,-\theta_{0}}$ can be zero only when the atom polarization vector is in the intersection of the two relevant vector spaces, which can be shown to be the one-dimensional complex vector space generated by $-\hat{\mathbf{x}}+$ $i \cos \theta_{0} \hat{\mathbf{z}}$. In other words, in the very special case in which the atomic polarization state satisfies

$$
\boldsymbol{\gamma} \sim-\hat{\mathbf{x}}+i \cos \theta_{0} \hat{\mathbf{z}}
$$

the lateral force may vanish. This effect can be attributed to the spin-momentum locking of the SPP [55]. Note that by tuning the bias magnetic field it is possible to adjust the value of $\theta_{0}$ and thereby guarantee that the lateral force does not vanish for any orientation of a given atom.

As an example, consider the case of a linearly polarized atom. Let us introduce the polarization factor $g_{\gamma}=$ $\frac{1}{2}\left(\Gamma_{+, \theta_{0}}+\Gamma_{+,-\theta_{0}}\right)$ which depends uniquely on the orienta- tion of the atom. If the atom has a random orientation the force is determined by the orientational averaging of the polarization factor $\left\langle g_{\gamma}\right\rangle$. With the rough approximation $\left\langle g_{\gamma}\right\rangle \approx \frac{1}{3}\left(g_{\hat{\mathbf{x}}}+g_{\hat{\mathbf{y}}}+g_{\hat{\mathbf{z}}}\right)$, we find that $\left\langle g_{\gamma}\right\rangle \approx \frac{2}{3}$. A detailed analysis shows that this result is actually exact, i.e., the orientational averaging of the polarization factor for a linearly polarized atom is precisely $\left\langle g_{\gamma}\right\rangle=\frac{2}{3}$, independent of the value of $\theta_{0}$.

\section{NUMERICAL EXAMPLES}

To illustrate the application of the developed theory, first we discuss the validity of the quasistatic solution. In general, one may expect that it should hold when the atom-interface distance is much smaller than the wavelength $\left(d \ll 2 \pi c / \omega_{p}\right.$ and $\left.d \ll 2 \pi c / \omega_{0}\right)$, so that the effects of time retardation are negligible. In addition, the quasistatic calculation assumes that the material absorption is negligible. In all the numerical examples presented below it is supposed that the dipole moment is along the $z$ direction, so that $\mathscr{F}_{y}=0$.

Figure 4(a) compares the exact solution for the lateral force with the quasistatic approximation (41), showing how the normalized force varies with the distance to the interface. For small distances $d \omega_{p} / c<0.3$ the normalized force is constant, confirming the $1 / d^{4}$ power law. For larger separations the quasistatic solution loses accuracy, and the force follows a different power law. However, it should be noted that for large $d$ the force is also much weaker (the coupling to the SPPs is weaker) and hence it is not so relevant.

Figure 4(b) shows a comparison between the two calculation methods when the distance is kept fixed and the atomic transition frequency $\omega_{0}$ is varied. There is an excellent agreement between the two solutions, further validating the quasistatic approximation. The small discrepancy between the two methods for $\omega \approx \omega_{-}$and $\omega \approx \omega_{+}$is attributed in part to the fact that the exact calculation includes the effect of material absorption $\left(\Gamma=0.015 \omega_{p}\right)$. As discussed in detail in [39], the lateral force sign depends on $\omega_{0}$. Furthermore, if the bias magnetic field is flipped $\left(\omega_{c}<0\right)$ the sign of the lateral force is also flipped. 

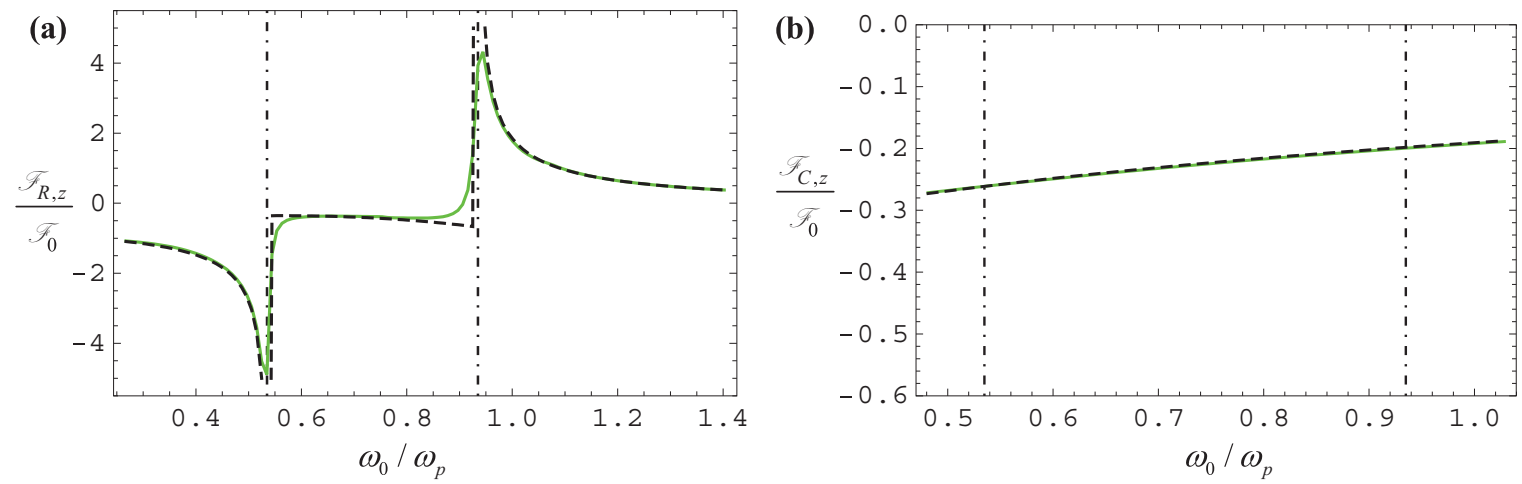

FIG. 5. Normal component of the (a) resonant force $\mathscr{F}_{R}$ and (b) nonresonant force $\mathscr{F}_{C}$ as a function of the atom transition frequency $\omega_{0}$ for $\omega_{c}=0.4 \omega_{p}$ and $d=0.01 c / \omega_{p}$. (Green solid lines) Exact result for a plasma collision frequency $\Gamma=0.015 \omega_{p}$; (black dashed lines) quasistatic result. The vertical grid lines mark the points $\omega=\omega_{ \pm}$.

Next, we focus on the normal component of the force. Figure 5 shows a comparison of the resonant part [Fig. 5(a)] and nonresonant part [Fig. 5(b)] of the normal force calculated by the exact and quasistatic solutions as a function of atom transition frequency. Again, the quasistatic method agrees well with the exact solution, excepting that the quasistatic solution diverges at $\omega_{ \pm}$for the resonant part of the force, whereas the exact calculation provides a finite result. Consistent with the discussion in Sec. IVC, the resonant force amplitude in the range $\omega_{-}<\omega_{0}<\omega_{+}$is negligible as compared to the value of the force outside this interval. Furthermore, the amplitude of the nonresonant component of the force is typically at least one order of magnitude smaller than the resonant component. As it also happens for standard reciprocal materials, depending on the value of $\omega_{0}$ the sign of resonant component $\mathscr{F}_{R, z}$ can be either positive or negative, but the nonresonant term is always negative (attractive force). We would like to note that the debate about the correct form of the metal response for low temperatures and its implications on the thermal corrections of the Casimir force [56] does not affect our calculations of the nonresonant force component, since the theory does not include any thermal effects and gives the zero-temperature limit solution.

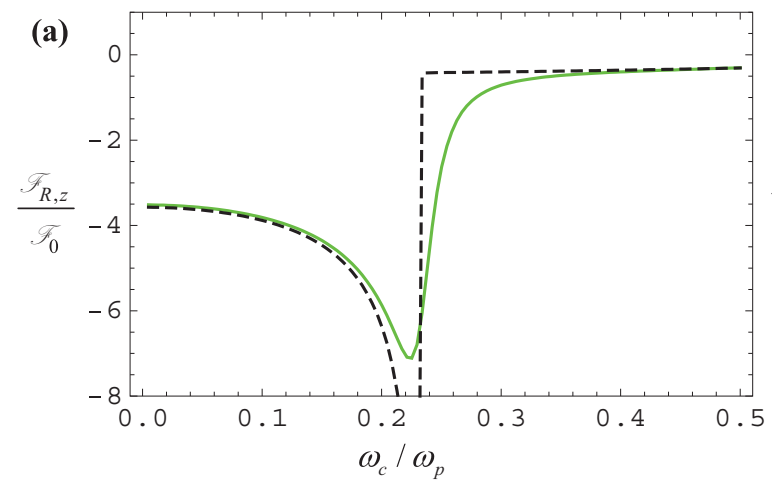

Figure 6 illustrates how the normal force varies with the plasma biasing strength. The nonresonant component of the force $\mathscr{F}_{C}$ is weakly sensitive to the magnetic bias. Different from the lateral force (which has odd symmetry), the vertical force components are even with respect to $\omega_{c}$ when the atom is polarized along the vertical direction. Hence, it is not possible to tune the sign of the normal force by changing the bias magnetic field.

Figure 7(a) shows a density plot of the total normal force (both resonant and nonresonant parts) for an excited atom $\left(\rho_{e e}=1\right)$ as a function of the magnetic bias and of the atomic transition frequency calculated by the exact solution. In this panel, for a given cyclotron frequency $\omega_{c}$ the bright areas correspond to $\omega_{0}=\omega_{ \pm}$, where the peak of the normal force occurs. It should be noted that for $\omega_{0}=\omega_{+}$and $\omega_{0}=\omega_{-}$the sign of the force changes. Furthermore, consistent with (47) it can be seen that a large force is achievable at low bias.

Next, we use (31) as a simplified model of InSb with $\omega_{p} / 2 \pi \approx 4.9 \mathrm{THz}$, cyclotron frequency in the range of $0.25 \omega_{p}-\omega_{p}$ for a bias field of $1-4$ Tesla, and collision frequency $\Gamma / 2 \pi=0.5 \mathrm{THz}$ [51]. For simplicity, we disregard the contribution of bound electrons to the permittivity response of InSb, and in particular its static permittivity is taken identical to unity. Figure 7(b) shows the effect of loss on the total normal

FIG. 6. Normal component of the (a) resonant force $\mathscr{F}_{R}$ and (b) nonresonant force $\mathscr{F}_{C}$ as a function of the cyclotron frequency $\omega_{c}$ for $\omega_{0}=0.6 \omega_{p}$ and $d=0.01 c / \omega_{p}$. (Green solid lines) Exact result for a plasma collision frequency $\Gamma=0.015 \omega_{p}$; (black dashed lines) quasistatic result. 
(a)

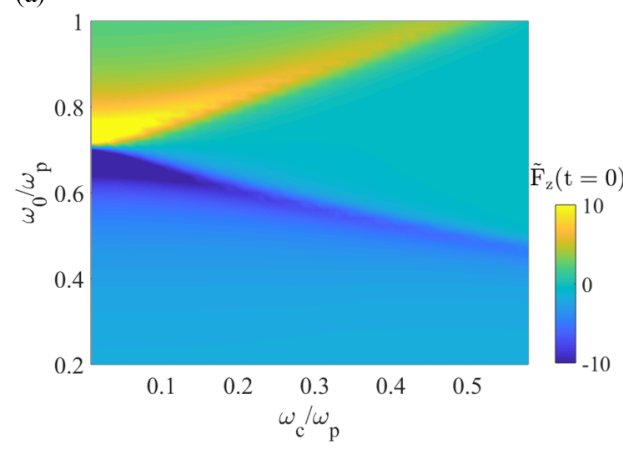

(b)

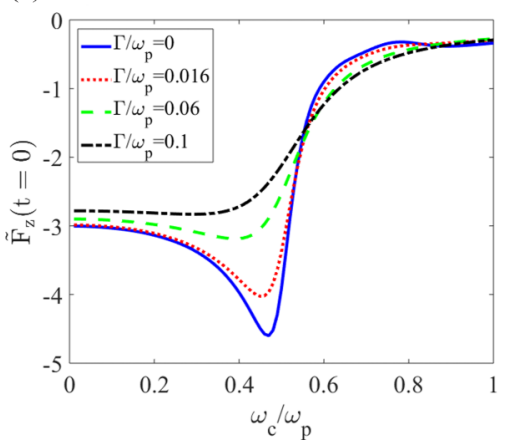

(c)

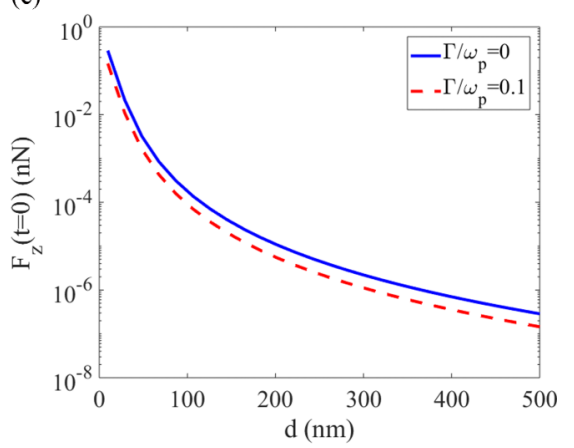

FIG. 7. (a) Density plot of the total normalized normal force $\left(\tilde{\mathrm{F}}_{z}=\mathscr{F}_{z} / \mathscr{F}_{0}\right)$ as a function of atom transition frequency and bias strength for $d=0.01 c / \omega_{p}$. (b) Normal force as a function of the bias strength for an atom located at $d=0.01 c / \omega_{p}$ above a biased plasma (InSb-type material) with $\omega_{p} / 2 \pi=4.9 \mathrm{THz}$ and $\omega_{0} / \omega_{p}=0.5$ for different values of collision frequency. (c) Normal force when the atom-interface distance is varied for $\omega_{0} / \omega_{p}=0.93$ and $\omega_{c} / \omega_{p}=0.4$.

component of the force, when the collision frequency varies from zero (lossless) to $0.5 \mathrm{THz}$. Even in the lossy case, there still exists considerable force applied to the atom. In general, the effect of loss is relatively mild, but near the resonant points $\omega_{0}=\omega_{ \pm}$the system is more sensitive to material absorption. Figure 7(c) shows the normal force as a function of atom-InSb distance for lossless and lossy InSb for a Rydberg atom [57] having $\gamma=7900 \mathrm{D}$ calculated by the exact solution. As seen, a significant force persists even in the lossy case.

\section{CONCLUSION}

We have investigated the Casimir-Polder force on an excited atom in a general (nonreciprocal, bianisotropic, and dispersive) electromagnetic environment under the Markov approximation. The force is shown to have resonant and nonresonant contributions, and we obtain explicit expressions for the quantum optical force in terms of the system Green function. We have shown that a two-level atom interacting with a topological gyrotropic material enables exotic lightmatter interactions, such as a lateral recoil force in a laterally invariant, homogeneous system with a sign independent of the orientation and polarization of the atom [39]. Furthermore, the strength of both the lateral and normal components of the force is highly sensitive to the bias, and the sign of the lateral force can also be externally controlled. In contrast, the nonresonant Casimir-Polder force in the ground state is little affected by the static magnetic field. To enable physical insight into the phenomena, we have presented simple analytical expressions for the quasistatic force, showing that surface plasmons play a dominant role in these fluctuation-induced forces. Remarkably, we find that when the atomic transition frequency matches either the SPP resonances $\omega_{-}$or $\omega_{+}$the resonant components of the force (both lateral and normal) are greatly enhanced, and in the quasistatic case (with no time retardation) and in the absence of material loss the force diverges. Furthermore, this phenomenon persists even for a weak magnetic bias [39]. The material absorption damps somewhat the strength of the force but the effect appears to remain sufficiently strong to allow for an experimental verification. We also present a Sommerfeld integral representation for the Green function for a gyrotropic material half-space.

\section{ACKNOWLEDGMENTS}

The authors gratefully acknowledge discussions with S. Buhmann. This work was partially funded by Fundação para a Ciência e a Tecnologia under Project No. PTDC/EEITEL/4543/2014 and by Instituto de Telecomunicações under Project No. UID/EEA/50008/2013. M.S. thanks the CNRS and the group Theory of Light-Matter and Quantum Phenomena of the Laboratoire Charles Coulomb for hospitality during his stay in Montpellier.

\section{APPENDIX A: MODAL EXPANSION OF THE GREEN FUNCTION}

We introduce a frequency domain Green function $\mathbf{G}\left(\mathbf{r}, \mathbf{r}_{0}\right)$ defined as a six-tensor of the classical electric and magnetic dyadic Green function,

$$
\mathbf{G}=\left(\begin{array}{ll}
\mathbf{G}_{\mathrm{EE}} & \mathbf{G}_{\mathrm{EH}} \\
\mathbf{G}_{\mathrm{HE}} & \mathbf{G}_{\mathrm{HH}}
\end{array}\right),
$$

such that

$$
\mathbf{N} \cdot \mathbf{G}=\omega \mathbf{M} \cdot \mathbf{G}+i \mathbf{I} \delta\left(\mathbf{r}-\mathbf{r}_{0}\right),
$$

where $\mathbf{r}$ is the observation point, $\mathbf{r}_{0}$ is the source point, and

$$
\mathbf{N}=\left(\begin{array}{cc}
\mathbf{0} & i \nabla \times \mathbf{I}_{3 \times 3} \\
-i \nabla \times \mathbf{I}_{3 \times 3} & \mathbf{0}
\end{array}\right)
$$

The material matrix $\mathbf{M}=\mathbf{M}(\mathbf{r}, \omega)$ determines the electromagnetic properties of the environment, which in general may be a bianisotropic nonreciprocal structure. In the limit of no loss, it is possible to expand the Green function into the natural eigenmodes $\mathbf{F}_{n \mathbf{k}}$ of the problem $[34,45,49]$,

$$
\mathbf{G}\left(\mathbf{r}, \mathbf{r}_{0}, \omega\right)=\sum_{n \mathbf{k}} \frac{i}{2\left(\omega_{n \mathbf{k}}-\omega\right)} \mathbf{F}_{n \mathbf{k}}(\mathbf{r}) \otimes \mathbf{F}_{n \mathbf{k}}^{*}\left(\mathbf{r}_{0}\right),
$$

with $\mathbf{F}_{n \mathbf{k}}\left(\mathbf{r}_{0}\right)$ normalized as in Eq. (7). The sum is over all the cavity modes, i.e., modes with positive, negative, and zero frequencies $\omega_{n \mathbf{k}}$. 
Taking into account that $[45,49]$

$$
\sum_{n \mathbf{k}} \frac{1}{2} \mathbf{F}_{n \mathbf{k}}(\mathbf{r}) \otimes \mathbf{F}_{n \mathbf{k}}^{*}\left(\mathbf{r}^{\prime}\right)=\mathbf{M}_{\infty}^{-1} \delta\left(\mathbf{r}-\mathbf{r}^{\prime}\right)
$$

with $\mathbf{M}_{\infty}=\lim _{\omega \rightarrow \infty} \mathbf{M}(\mathbf{r}, \omega)$, it follows that

$$
\begin{aligned}
\mathbf{G} & =\sum_{n \mathbf{k}} \frac{1}{2} \frac{i \omega_{n \mathbf{k}}}{\left(\omega_{n \mathbf{k}}-\omega\right) \omega} \mathbf{F}_{n \mathbf{k}}(\mathbf{r}) \otimes \mathbf{F}_{n \mathbf{k}}^{*}\left(\mathbf{r}_{0}\right)-\frac{i}{\omega} \mathbf{M}_{\infty}^{-1} \delta\left(\mathbf{r}-\mathbf{r}_{0}\right) \\
= & \sum_{\omega_{n \mathbf{k}}>0} \frac{i \omega_{n \mathbf{k}}}{2 \omega}\left(\frac{1}{\omega_{n \mathbf{k}}-\omega} \mathbf{F}_{n \mathbf{k}}(\mathbf{r}) \otimes \mathbf{F}_{n \mathbf{k}}^{*}\left(\mathbf{r}_{0}\right)\right. \\
& \left.+\frac{1}{\omega_{n \mathbf{k}}+\omega} \mathbf{F}_{n \mathbf{k}}^{*}(\mathbf{r}) \otimes \mathbf{F}_{n \mathbf{k}}\left(\mathbf{r}_{0}\right)\right)-\frac{i}{\omega} \mathbf{M}_{\infty}^{-1} \delta\left(\mathbf{r}-\mathbf{r}_{0}\right) .
\end{aligned}
$$

In the second identity we used the fact that because of the reality of the electromagnetic field the eigenmodes with negative frequencies can be linked to the eigenmodes with positive frequencies by a complex conjugation, $\mathbf{G}^{*}\left(\mathbf{r}, \mathbf{r}_{0}, \omega\right)=$ $\mathbf{G}\left(\mathbf{r}, \mathbf{r}_{0},-\omega^{*}\right)$, and we assume $\omega \in \mathbb{R}$. For future reference, we decompose the Green function as $\mathbf{G}=\mathbf{G}^{+}+\mathbf{G}^{-}+$ $\frac{1}{i \omega} \mathbf{M}_{\infty}^{-1} \delta\left(\mathbf{r}-\mathbf{r}_{0}\right)$, where

$$
\begin{aligned}
& (-i \omega) \mathbf{G}^{+}=\sum_{\omega_{n \mathbf{k}}>0} \frac{\omega_{n \mathbf{k}}}{2} \frac{1}{\omega_{n \mathbf{k}}-\omega} \mathbf{F}_{n \mathbf{k}}(\mathbf{r}) \otimes \mathbf{F}_{n \mathbf{k}}^{*}\left(\mathbf{r}_{0}\right), \\
& (-i \omega) \mathbf{G}^{-}=\sum_{\omega_{n \mathbf{k}}>0} \frac{\omega_{n \mathbf{k}}}{2} \frac{1}{\omega_{n \mathbf{k}}+\omega} \mathbf{F}_{n \mathbf{k}}^{*}(\mathbf{r}) \otimes \mathbf{F}_{n \mathbf{k}}\left(\mathbf{r}_{0}\right)
\end{aligned}
$$

are the positive and negative frequency parts of the Green function, respectively.

\section{APPENDIX B: GREEN FUNCTION FOR A GYROTROPIC MATERIAL HALF-SPACE}

Here, we derive the electric Green dyadic $\mathbf{G}_{\mathrm{EE}}$ for the case of a gyrotropic material half-space. As discussed in Sec. II C, the vector $\mathbf{E}=-i \omega \mathbf{G}_{\mathrm{EE}} \cdot \boldsymbol{\gamma}$ gives the field emitted by a classical dipole with electric dipole moment $\boldsymbol{\gamma}$. Hence, $\mathbf{G}_{\mathrm{EE}}$ can be found from the field radiated by a generic dipole.

To calculate $\mathbf{E}$, we note that the electromagnetic field in the region $z>0$ (vacuum) is the superposition of the primary field $\left(\mathbf{E}^{\mathrm{p}}\right)$ and the scattered field $\left(\mathbf{E}^{\mathrm{s}}\right)$. The primary field is given by
$\mathbf{E}^{\mathrm{p}}=\left(\nabla \nabla+k_{0}^{2} \mathrm{I}\right)\left(\boldsymbol{\gamma} / \varepsilon_{0}\right) \Phi_{0}$ where

$$
\Phi_{0}=\frac{e^{i k_{0}\left|\mathbf{r}-\mathbf{r}_{0}\right|}}{4 \pi\left|\mathbf{r}-\mathbf{r}_{0}\right|}=\iint d k_{x} d k_{y} \frac{e^{-\gamma_{0}|z-d|}}{2 \gamma_{0}(2 \pi)^{2}} e^{i\left(k_{x} x+k_{y} y\right)}
$$

is the Hertz potential, $k_{0}=\omega_{0} / c$, and $\gamma_{0}=\sqrt{{k_{x}}^{2}+k_{y}{ }^{2}-k_{0}^{2}}$. Without loss of generality, it is assumed that the source point is $\mathbf{r}_{0}=(0,0, d)$.

Following [58], the scattered electric field above the interface can be written as

$$
\mathbf{E}^{s}=\frac{1}{(2 \pi)^{2}} \iint d k_{x} d k_{y} e^{i \mathbf{k}_{\|} \cdot \mathbf{r}} \frac{e^{-\gamma_{0}(d+z)}}{2 \gamma_{0}} \mathbf{C}\left(\omega, \mathbf{k}_{\|}\right) \cdot \frac{\gamma}{\varepsilon_{0}},
$$

where

$$
\begin{aligned}
\mathbf{C}\left(\omega, \mathbf{k}_{\|}\right)= & \left(\mathbf{I}_{\|}+\hat{\mathbf{z}} \frac{i \mathbf{k}_{\|}}{\gamma_{0}}\right) \cdot \mathbf{R}\left(\omega, \mathbf{k}_{\|}\right) \\
& \cdot\left(i \gamma_{0} \mathbf{k}_{\|} \hat{\mathbf{z}}+k_{0}^{2} \mathbf{I}_{\|}-\mathbf{k}_{\|} \mathbf{k}_{\|}\right)
\end{aligned}
$$

with $\mathbf{I}_{\|}=\hat{\mathbf{x}} \hat{\mathbf{x}}+\hat{\mathbf{y}} \hat{\mathbf{y}}$ and $\mathbf{k}_{\|}=k_{x} \hat{\mathbf{x}}+k_{y} \hat{\mathbf{y}}$. Here, $\mathbf{R}\left(\omega, k_{x}, k_{y}\right)$ is a $2 \times 2$ reflection matrix that relates the tangential (to the interface) components $x$ and $y$ of the reflected electric field to the corresponding $x$ and $y$ components of the incident field, $\left(\begin{array}{c}E_{x}^{\mathrm{s}} \\ E_{y}^{\mathrm{s}}\end{array}\right)=\mathbf{R}\left(\omega, k_{x}, k_{y}\right) \cdot\left(\begin{array}{c}E_{x}^{\mathrm{inc}} \\ E_{y}^{\text {inc }}\end{array}\right)$ for the case of plane wave incidence. Since (B2) holds for a generic electric dipole, it is straightforward to verify that $\mathbf{G}_{\mathrm{EE}}$ has the decomposition discussed in the main text, with the scattering part of the Green function given by (29).

To determine an explicit formula for $\mathbf{R}$, it is assumed that the region $z<0$ is filled with a gyrotropic material with dielectric function given by (28). The incident plane wave travels in the isotropic material region.

Evidently, the fields depend on $x$ and $y$ as $e^{i k_{x} x} e^{i k_{y} y}$. In the region $z<0$ they can be written as a superposition of two plane waves of the bulk gyrotropic medium with wave vector $\mathbf{k}_{i}=$ $\mathbf{k}_{t, i}+k_{y} \hat{\mathbf{y}}$, with $\mathbf{k}_{t, i}=k_{x} \hat{\mathbf{x}}+k_{z, i} \hat{\mathbf{z}}(i=1,2)$. The subscript " $t$ " indicates that a certain vector component is perpendicular to the $y$ direction, which corresponds the direction of the bias magnetic field. Setting $k_{z, i}=-i \gamma_{z, i}$ such that $\operatorname{Re}\left(\gamma_{z, i}\right)>0$, the bulk mode dispersion is [47]

$$
\begin{aligned}
\gamma_{z, i}^{2}= & k_{x}^{2}-\frac{1}{2 \varepsilon_{t}}\left[\left(\varepsilon_{t}\left(\varepsilon_{t}+\varepsilon_{a}\right)-\varepsilon_{g}^{2}\right) k_{0}^{2}-\left(\varepsilon_{a}+\varepsilon_{t}\right) k_{y}^{2}\right] \\
& \pm \frac{1}{2 \varepsilon_{t}} \sqrt{\left[\left(\varepsilon_{t}\left(\varepsilon_{t}+\varepsilon_{a}\right)-\varepsilon_{g}^{2}\right) k_{0}^{2}-\left(\varepsilon_{a}+\varepsilon_{t}\right) k_{y}^{2}\right]^{2}-4 \varepsilon_{t}\left[\left(\varepsilon_{t}^{2}-\varepsilon_{g}^{2}\right) \varepsilon_{a} k_{0}^{4}-2 \varepsilon_{t} \varepsilon_{a} k_{y}^{2} k_{0}^{2}+\varepsilon_{a} k_{y}^{4}\right]}
\end{aligned}
$$

Each of these possible solutions is associated with a plane wave. For a plane wave superposition, the electric field is of the form [47],

$$
\begin{aligned}
\mathbf{E}= & \left(\Delta_{1} \mathbf{k}_{1} \times \hat{\mathbf{y}}+\mathbf{k}_{t, 1}+\theta_{1} k_{y} \hat{\mathbf{y}}\right) A_{1} e^{\gamma_{z, 1} z} \\
& +\left(\Delta_{2} \mathbf{k}_{2} \times \hat{\mathbf{y}}+\mathbf{k}_{t, 2}+\theta_{2} k_{y} \hat{\mathbf{y}}\right) A_{2} e^{\gamma_{z, 2} z}
\end{aligned}
$$

where the variation along $x$ and $y$ is omitted, $A_{i}(i=1,2)$ are expansion coefficients, and

$$
\Delta_{i}=\frac{i \varepsilon_{g} k_{0}^{2}}{k_{0}^{2} \varepsilon_{t}-\left(k_{y}^{2}+k_{t, i}^{2}\right)}, \quad \theta_{i}=\frac{-k_{t, i}^{2}}{k_{0}^{2} \varepsilon_{a}-k_{t, i}^{2}} .
$$

The magnetic field can be found from (B5), taking into account that for each plane wave $\mathbf{H}=\mathbf{k} \times \mathbf{E} / \omega \mu_{0}$. Using (B5) and the 
magnetic field expression, the expansion coefficients can be eliminated, leading to

$$
\left(\begin{array}{c}
\eta_{0} H_{y} \\
-\eta_{0} H_{x}
\end{array}\right)=-\mathbf{Y}_{g} \cdot\left(\begin{array}{c}
E_{y} \\
E_{x}
\end{array}\right)
$$

with $\eta_{0}$ the vacuum impedance and

$$
\begin{aligned}
\mathbf{Y}_{g}= & \left(\begin{array}{cc}
\frac{\Delta_{1} k_{t, 1}^{2}}{k_{0}} & \frac{\Delta_{2} k_{t, 2}^{2}}{k_{0}} \\
\frac{\Delta_{1} k_{x} k_{y}+i \gamma_{z, 1}\left(\theta_{1}-1\right) k_{y}}{k_{0}} & \frac{\Delta_{2} k_{x} k_{y}+i \gamma_{z, 2}\left(\theta_{2}-1\right) k_{y}}{k_{0}}
\end{array}\right) \\
& \cdot\left(\begin{array}{cc}
k_{x}+i \gamma_{z, 1} \Delta_{1} & k_{x}+i \gamma_{z, 2} \Delta_{2} \\
\theta_{1} k_{y} & \theta_{2} k_{y}
\end{array}\right)^{-1} .
\end{aligned}
$$

Note that the considered field distribution corresponds to a wave that propagates towards the $-z$ direction in the gyrotropic material. Likewise, it is possible to show that for a wave that propagates in the isotropic dielectric (air region) in the $\pm z$ direction the fields satisfy $\left(\begin{array}{c}\eta_{0} H_{y} \\ -\eta_{0} H_{x}\end{array}\right)= \pm \mathbf{Y}_{0} \cdot\left(\begin{array}{c}E_{x} \\ E_{y}\end{array}\right)$ with

$$
\mathbf{Y}_{0}=\frac{1}{i k_{0} \gamma_{0}}\left(\begin{array}{cc}
-\gamma_{0}^{2}+k_{x}^{2} & k_{x} k_{y} \\
k_{x} k_{y} & -\gamma_{0}^{2}+k_{y}^{2}
\end{array}\right)
$$

where $\gamma_{0}^{2}=k_{x}^{2}+k_{y}^{2}-k_{0}^{2}$.

It is now straightforward to obtain the reflection matrix $\mathbf{R}$. Noting that the field in the region $z>0$ is a superposition of the incident and reflected waves and that the field in the region $z<0$ is of the form (B5), it follows, imposing the continuity of the tangential fields at the interface, that $\mathbf{Y}_{0} \cdot(-\mathbf{1}+\mathbf{R})=$ $-\mathbf{Y}_{g}(\mathbf{1}+\mathbf{R})$. From here we obtain the desired result,

$$
\mathbf{R}=\left(\mathbf{Y}_{0}+\mathbf{Y}_{g}\right)^{-1} \cdot\left(\mathbf{Y}_{0}-\mathbf{Y}_{g}\right) .
$$

\section{APPENDIX C: EXACT DISPERSION EQUATION FOR THE SURFACE PLASMONS}

Here, we derive the exact dispersion equation for the surface plasmons assuming that the region $z>0$ is free-space and that the region $z<0$ is a magnetized gyrotropic plasma. It is supposed that the interface between the two regions is perfectly smooth, and hence possible contributions to the SPPs dispersion due to surface roughness are disregarded.

The fields in the two regions can be expanded into evanescent plane waves. In particular, in the bulk gyrotropic medium the modes can be written as a superposition of two plane waves with the $z$ propagation factor defined as in (B4) and the electric field given by (B5). The associated magnetic field in the region $z<0$ can be found using $\mathbf{H}=\mathbf{k} \times \mathbf{E} / \omega \mu_{0}$ for each plane wave term.

The fields in the vacuum region $(z>0)$ can be expanded as

$$
\begin{aligned}
\mathbf{E} & =-\left[B_{1} \mathbf{k}_{0} \times \hat{\mathbf{z}}+B_{2} \mathbf{k}_{0} \times\left(\mathbf{k}_{0} \times \hat{\mathbf{z}}\right)\right] e^{-\gamma_{0} z} \\
\omega \mu_{0} \mathbf{H} & =-\left[B_{1} \mathbf{k}_{0} \times\left(\mathbf{k}_{0} \times \hat{\mathbf{z}}\right)-B_{2} \frac{\omega^{2}}{c^{2}}\left(\mathbf{k}_{0} \times \hat{\mathbf{z}}\right)\right] e^{-\gamma_{0} z},
\end{aligned}
$$

with $\mathbf{k}_{0}=k_{x} \hat{\mathbf{x}}+k_{y} \hat{\mathbf{y}}+i \gamma_{0} \hat{\mathbf{z}}$ and $\gamma_{0}=\sqrt{k_{x}^{2}+k_{y}^{2}-\omega^{2} / c^{2}}$. By matching the tangential electromagnetic fields at the interface $(z=0)$ we arrive at the following system of equations,

$$
\begin{gathered}
\left(\begin{array}{cccc}
k_{x}+i \gamma_{z, 1} \Delta_{1} & k_{x}+i \gamma_{z, 2} \Delta & k_{y} & k_{x} i \gamma_{0} c / \omega \\
\theta_{1} k_{y} & \theta_{2} k_{y} & -k_{x} & k_{y} i \gamma_{0} c / \omega \\
\Phi_{1} & \Phi_{2} & k_{x} i \gamma_{0} & -k_{y} \omega / c \\
-\Delta_{1} k_{t, 1}^{2} & -\Delta_{2} k_{t, 2}^{2} & k_{y} i \gamma_{0} & k_{x} \omega / c
\end{array}\right) \\
\cdot\left(\begin{array}{c}
A_{1} \\
A_{2} \\
B_{1} \\
B_{2} \frac{\omega}{c}
\end{array}\right)=\mathbf{0}_{4 \times 1},
\end{gathered}
$$

where $\theta_{i}, \Delta_{i}$, and $\gamma_{z, i}$ are defined in Appendix B and $\Phi_{i}=$ $\Delta_{i} k_{x} k_{y}+i \gamma_{z, i}\left(\theta_{i}-1\right) k_{y},(i=1,2)$. Setting the determinant of the matrix equal to zero leads to the exact SPP dispersion equation.
[1] H. B. G. Casimir and D. Polder, The Influence of Retardation on the London-van der Waals Forces, Phys. Rev. 73, 360 (1948).

[2] I. E. Dzyaloshinskii, E. M. Lifshitz, and L. P. Pitaevskii, The general theory of van der Waals forces, Adv. Phys. 10, 165 (1961).

[3] G. S. Agarwal, Quantum electrodynamics in the presence of dielectrics and conductors. I. Electromagnetic-field response functions and black-body fluctuations in finite geometries, Phys. Rev. A 11, 243 (1975).

[4] A. M. Marvin and F. Toigo, van der Waals interaction between a point particle and a metallic surface. I. Theory, Phys. Rev. A 25, 782 (1982).

[5] J. M. Wylie and J. E. Sipe, Quantum electrodynamics near an interface, Phys. Rev. A 30, 1185 (1984).

[6] J. M. Wylie and J. E. Sipe, Quantum electrodynamics near an interface. II, Phys. Rev. A 32, 2030 (1985).
[7] P. W. Milonni and M.-L. Shih, Source theory of the Casimir force, Phys. Rev. A 45, 4241 (1992).

[8] M. Fichet, F. Schuller, D. Bloch, and M. Ducloy, van der Waals interactions between excited-state atoms and dispersive dielectric surfaces, Phys. Rev. A 51, 1553 (1995).

[9] M. Boustimi, J. Baudon, P. Candori, and J. Robert, van der Waals interaction between an atom and a metallic nanowire, Phys. Rev. B 65, 155402 (2002).

[10] C. Henkel, K. Joulain, J.-P. Mulet, and J.-J. Greffet, Radiation forces on small particles in thermal near fields, J. Opt. A: Pure Appl. Opt. 4, S109 (2002).

[11] C.-H. Wu, C.-I. Kuo, and L. H. Ford, Fluctuations of the retarded van der Waals force, Phys. Rev. A 65, 062102 (2002).

[12] S. Y. Buhmann, L. Knoll, D.-G. Welsch, and H. T. Dung, Casimir-Polder forces: A nonperturbative approach, Phys. Rev. A 70, 052117 (2004). 
[13] M. Antezza, L. P. Pitaevskii, and S. Stringari, New Asymptotic Behavior of the Surface-Atom Force out of Thermal Equilibrium, Phys. Rev. Lett. 95, 113202 (2005).

[14] J. M. Obrecht, R. J. Wild, M. Antezza, L. P. Pitaevskii, S. Stringari, and E. A. Cornell, Measurement of the temperature dependence of the Casimir-Polder force, Phys. Rev. Lett. 98, 063201 (2007).

[15] S. Y. Buhmann and S. Scheel, Thermal Casimir versus CasimirPolder Forces: Equilibrium and Nonequilibrium Forces, Phys. Rev. Lett. 100, 253201 (2008).

[16] S. Scheel, S. Y. Buhmann, C. Clausen, and P. Schneeweiss, Directional spontaneous emission and lateral Casimir-Polder force on an atom close to a nanofiber, Phys. Rev. A 92, 043819 (2015).

[17] S. Fuchs, F. Lindel, R. V. Krems, G. W. Hanson, M. Antezza, and S. Buhmann, Casimir-Lifshitz force for nonreciprocal media and applications to photonic topological insulators, Phys. Rev. A 96, 062505 (2017).

[18] F. Intravaia, C. Henkel, and M. Antezza, Fluctuation-induced forces between atoms and surfaces: The Casimir-Polder interaction, in Lecture Notes in Physics for a volume on Casimir Physics, edited by D. Dalvit, P. Milonni, D. Roberts, and F. da Rosa (Springer-Verlag, Berlin/Heidelberg, 2010)

[19] U. Leonhardt and T. G. Philbin, Quantum levitation by lefthanded metamaterials, New J. Phys. 9, 254 (2007).

[20] R. Zhao, J. Zhou, T. Koschny, E. N. Economou, and C. M. Soukoulis, Repulsive Casimir Force in Chiral Metamaterials, Phys. Rev. Lett. 103, 103602 (2009).

[21] M. G. Silveirinha and S. I. Maslovski, Comment on "Repulsive Casimir Force in Chiral Metamaterials", Phys. Rev. Lett. 105, 189301 (2010).

[22] F. S. S. Rosa, D. A. R. Dalvit, and P. W. Milonni, Casimir-Lifshitz Theory and Metamaterials, Phys. Rev. Lett. 100, 183602 (2008).

[23] V. Yannopapas and N. V. Vitanov, First-Principles Study of Casimir Repulsion in Metamaterials, Phys. Rev. Lett. 103, 120401 (2009).

[24] M. G. Silveirinha, Casimir interaction between metal-dielectric metamaterial slabs: Attraction at all macroscopic distances, Phys. Rev. B 82, 085101 (2010).

[25] M. G. Silveirinha and S. I. Maslovski, Physical restrictions on the Casimir interaction of metal-dielectric metamaterials: An effective medium approach, Phys. Rev. A, 82, 052508 (2010).

[26] S. I. Maslovski and M. G. Silveirinha, Ultralong-range CasimirLifshitz forces mediated by nanowire materials, Phys. Rev. A 82, 022511 (2010).

[27] S. I. Maslovski and M. G. Silveirinha, Mimicking Boyer's Casimir Repulsion with a Nanowire Material, Phys. Rev. A 83, 022508 (2011).

[28] A. G. Grushin and A. Cortijo, Tunable Casimir Repulsion with Three-Dimensional Topological Insulators, Phys. Rev. Lett. 106, 020403 (2011).

[29] L. Lu, J. D. Joannopoulos, and M. Soljacic, Topological photonics, Nat. Photon. 8, 821 (2014).

[30] L. Lu, J. D. Joannopoulos, and M. Soljacic, Topological states in photonic systems, Nat. Phys. 12, 626 (2016).

[31] F. D. M. Haldane and S. Raghu, Possible Realization of Directional Optical Waveguides in Photonic Crystals with Broken Time-Reversal Symmetry, Phys. Rev. Lett. 100, 013904 (2008).
[32] S. Raghu and F. D. M. Haldane, Analogs of quantum-Hall-effect edge states in photonic crystals, Phys. Rev. A 78, 033834 (2008).

[33] Z. Wang, Y. Chong, J. D. Joannopoulos, and M. Soljacic, Observation of unidirectional backscattering immune topological electromagnetic states, Nature (London) 461, 772 (2009).

[34] M. G. Silveirinha, Chern Invariants for Continuous Media, Phys. Rev. B 92, 125153 (2015).

[35] M. G. Silveirinha, Bulk-edge correspondence for topological photonic continua, Phys. Rev. B 94, 205105, (2016).

[36] S. A. H. Gangaraj, M. G. Silveirinha, and G. W. Hanson, Berry Phase, Berry Connection, and Chern Number for a Continuum Bianisotropic Material from a Classical Electromagnetics Perspective, IEEE J. Multiscale Multiphys. Comput. Techn. 2, 3 (2017).

[37] M. G. Silveirinha, PTD Symmetry Protected Scattering Anomaly in Optics, Phys. Rev. B 95, 035153 (2017).

[38] S. A. Hassani Gangaraj, G. W. Hanson, and M. Antezza, Robust entanglement with three-dimensional nonreciprocal photonic topological insulators, Phys. Rev. A 95, 063807 (2017).

[39] S. A. H. Gangaraj, G. W. Hanson, M. Antezza, and M. G. Silveirinha, Spontaneous lateral atomic recoil force close to a photonic topological material, arXiv:1711.4939.

[40] F. J. Rodríguez-Fortuño, G. Marino, P. Ginzburg, D. O’Connor, A. Martínez, G. A. Wurtz, and A. V. Zayats, Near-Field Interference for the Unidirectional Excitation of Electromagnetic Guided Modes, Science 340, 328 (2013).

[41] S. B. Wang and C. T. Chan, Lateral optical force on chiral particles near a surface, Nat. Commun. 5, 3307 (2014).

[42] F. J. Rodríguez-Fortuño, N. Engheta, A. Martínez, and A. V. Zayats, Lateral forces on circularly polarizable particles near a surface, Nat. Commun. 6, 8799 (2015).

[43] I. S. Nefedov and J. M. Rubi, Lateral-drag propulsion forces induced by anisotropy, Sci. Rep. 7, 6155 (2017).

[44] The decay rate $\Gamma_{e g}$ is not included in the formula but its effect is negligible because the integration is over the imaginary axis and for typical atomic systems $\Gamma_{e g} \ll \omega_{0}$.

[45] S. Lannebere and M. G. Silveirinha, Negative spontaneous emission by a moving two-level atom, J. Opt. 19, 014004 (2017).

[46] J. P. Gordon and A. Ashkin, Motion of atoms in a radiation trap, Phys. Rev. A 21, 1606, (1980).

[47] M. G. Silveirinha, Topological angular momentum and radiative heat transport in closed orbits, Phys. Rev. B 95, 115103 (2017).

[48] N. A. R. Bhat and J. E. Sipe, Hamiltonian treatment of the electromagnetic field in dispersive and absorptive structured media, Phys. Rev. A 73, 063808 (2006).

[49] M. G. Silveirinha, Modal expansions in dispersive material systems with application to quantum optics and topological photonics, in Advances in Mathematical Methods for Electromagnetics, edited by P. Smith and K. Kobayashi, arXiv: 1712.4272 .

[50] J. A. Bittencourt, Fundamentals of Plasma Physics, 3rd ed. (Springer-Verlag, New York, 2010).

[51] E. Palik, R. Kaplan, R. Gammon, H. Kaplan, R. Wallis, and J. Quinn, Coupled surface magnetoplasmon-optic-phonon polariton modes on InSb, Phys. Rev. B 13, 2497 (1976).

[52] E. Moncada-Villa, V. Fernandez-Hurtado, F. J. Garcia-Vidal, A. Garcia-Martin, and J. C. Cuevas, Magnetic field control of nearfield radiative heat transfer and the realization of highly tunable hyperbolic thermal emitters, Phys. Rev. B 92, 125418 (2015). 
[53] R. E. Camley, Nonreciprocal surface waves, Surf. Sci. Rep. 7, 103 (1987).

[54] A. R. Davoyan and N. Engheta, Theory of Wave Propagation in Magnetized Near-Zero-Epsilon Metamaterials: Evidence for One-Way Photonic States and Magnetically Switched Transparency and Opacity, Phys. Rev. Lett. 111, 257401 (2013).

[55] K. Y. Bliokh, F. J. Rodriguez-Fortuno, F. Nori, and A. V. Zayats, Spin—orbit interactions of light, Nat. Photon. 9, 796 (2015).
[56] A. O. Sushkov, W. J. Kim, D. A. R. Dalvit, and S. K. Lamoreaux, Observation of the thermal Casimir force, Nat. Phys. 7, 230 (2011).

[57] T. F. Gallagher, Rydberg atoms, Rep. Prog. Phys. 51, 143 (1988).

[58] M. G. Silveirinha, Optical Instabilities and Spontaneous Light Emission by Polarizable Moving Matter, Phys. Rev. X 4, 031013 (2014). 